\title{
THEORETICAL STUDIES IN NUCLEAR REACTIONS AND NUCLEAR STRUCTURE
}

\author{
A Progress Report for Research Initiated Under \\ D.O.E. Grant No. DE-FG05-87ER-40322
}

University of Maryland

Submitted May 1992

to the

U. S. Department of Energy

DISCLAIMER

Department of Physics

This report was prepared as an account of work sponsored by an agency of the United States Government. Neither the United States Government nor any agency thereof, nor any of their employees, makes any warranty, express or implied, or assumes any legal liability or responsibility for the accuracy, completeness, or usefulness of any information, apparatus, product, or process disclosed, or represents that its use would not infringe privately owned rights. Reference herein to any specific commercial product, process, or service by trade name, trademark, manufacturer, or otherwise does not necessarily constitute or imply its endorsement, recommendation, or favoring by the United States Government or any agency thereof. The views and opinions of authors expressed herein do not necessarily state or reflect those of the United States Government or any agency thereof.

\footnotetext{
College Park, Maryland 20742-4111
}

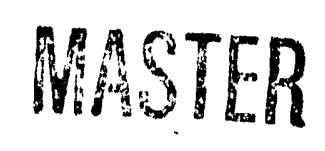




\section{Contents}

Introduction

$\begin{array}{lll}\text { I Hadrons in Nuclear Matter } & 7\end{array}$

A QCD Sum Rules and Nucleons in Nuclear Matter . . . . . . . . . 8

B QCD Condensates in Nuclear matter ................ 9

C Vector Mesons at Finite Temperature/Density from QCD Sum Rules . . . 10

D Nucleon in Nuclear Matter . . . . . . . . . . . . . . . . 12

E A New Approach to Dense Matter in the Nambu-Jona-Lasinio Model . . 13

F Passage of High-Energy Partons through a Quark-Gluon Plasma . . . . . . 14

G Coherent Photoproduction of the Neutral Pion as a Test of In-Medium Vector Meson Masses . . . . . . . . . . . . . . . . . 15

$\mathrm{H}$ The Axial Coupling in Nuclear Matter ............... 15

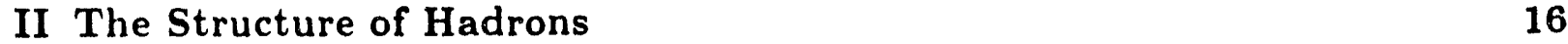

A QCD Sum Rules and The Sigma Term . . . . . . . . . . . . . 19

B Decuplet Baryon Electromagnetic Structure on the Lattice . . . . . . . . . 19

C The $\mathrm{N} \gamma \rightarrow \Delta$ Electromagnetic Transition in Lattice QCD . . . . . . . . . 20

D Effect of Instantons in the Chiral Confining Modei . . . . . . . . . . . . 20

E Relativistic Quark Model of $q q$ Mesons . . . . . . . . . . . . . . 23

F Nucleon Electromagnetic Polarizibilities in Chiral iłodels. . . . . . . . 26

G Chiral Corrections to Lattice Calculations of Charge Radii . . . . . . . . . 27

H Are There Pion Loops In Quenched QCD? . . . . . . . . . . . . . 27

I Hedgehog models and Chiral Perturbation Theory . . . . . . . . . 28

III Relativistic Nuclear Physics $\quad 28$

A Boost, recoil, and Wigner rotation effects on no-pair analyses of proton

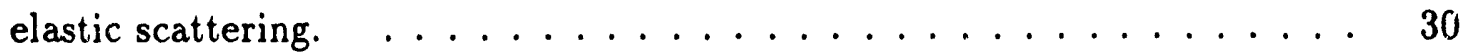


B Lectures on Relativistic Nuclear Physics . . . . . . . . . . . . . . 32

C Relativistic and Off-Shell Effects in Elastic Proton Scattering. . . . . . . 34

D Relativistic Analysis of the Deuteron and Elastic Electron-Deuteron Scattering 36

E Effective Interaction Based on the Relativistic IA2 Formalism . . . . . . 38

F Full Folding Optical Potential Analysis . . . . . . . . . . . . . . . 42

G Three Body Problem and Relativity . . . . . . . . . . . . . . . 44

IV Heavy Ion Dynamics and Related Processes 46

A Overview.......................... 46

B Leptonic Emissions from High-Z Heavy Ion Collisions . . . . . . . . . . 47

1 The Composite Particle ( $Q_{0}$ ?) Senario for the GSI Lines, and Bound Decays . . . . . . . . . . . . . . . . . . . 47

2 One Photon Decay of Composite $C\left(Q_{0}\right.$ ?) Particle . . . . . . . . 48

3 Bi-Positron (BiPE) Emittive Decay of $C\left(Q_{0} ?\right) \ldots \ldots$

4 Tri-Lepton (TriLE) Emittive Decay of $C\left(Q_{0}\right.$ ? $) \ldots \ldots \ldots$

5 Occurence of Bound Decays for U+Ta, Not for U+Th . . . . . . 49

6 Alleged Constraints on the Composite $\left(Q_{0}\right.$ ?) Particle Lifetime from Bhabha Scattering Data are False . . . . . . . . . . . . 50

$7 \quad$ Alleged Limits on $C\left(Q_{0}\right.$ ?) Lifetime from $\left(\mathrm{g}_{\mathrm{e}}-2\right)$ Apply Only to Elementary $\mathrm{X}_{0}$ Particles, Not to Composites . . . . . . . . 50

8 Fundamental Origins of Quadronium Binding Energies . . . . . . 51

C Theoretical Studies of Heavy Ion Dynamics . . . . . . . . . . . . 52

1 Permeation Current and $\bar{N}, \bar{Z}$ Drifts in Heavy Ion Collisions . . . 52

Nuclear Theory Group Personnel

$\begin{array}{ll}\text { Curricula Vita of Faculty } & 57\end{array}$

$\begin{array}{ll}\text { Research Publications } & 67\end{array}$ 


\section{Introduction}

Research in the Maryland Nuclear Theory Group focusses on problems in four basic areas of current relevance. They form the major divisions of the progress report as follows.

I. Hadrons in Nuclear Matter

II. The Structure of Hadrons

III. Relativistic Nuclear Physics

IV. Heavy Ion Dynamics and Related Processes

Publications and research progress in the past year are reported. The section on Hadrons in Nuclear Matter groups together research items which are aimed at exploring ways in which the properties of nucleons and the mesons which play a role in the nuclear force are modified in the nuclear medium. A very interesting result has been the finding that QCD sum rules supply a new insight into the decrease of the nucleon's mass in the nuclear medium. The quark condensate, which characterizes spontaneous chiral symmetry breaking of the the QCD vacuum, decreases in nuclear matter and this is responsible for the decrease of the nucleon's mass. The section on the Structure of Hadrons contains progress reports on our research aimed at understanding the structure of the nucleon. Widely different aproaches are being studied, e.g., lattice gauge calculations, QCD sum rules, quark-meson models with confinement and other hedgehog modtis. A major goal of this type of research is to develop appropriate links between nuclear physics and QCD. The section on Relativistic Nuclear Physics represents our continuing interest in developing an appropriate relativistic framework for nuclear dynamics. A Lorentz-invariant description of the nuclear force suggests a similar decrease of the nucleon's mass in the nuclear mediumn as has been found from QCD sum rules. Work in progress extends previous successes in elastic scattering to inelastic scattering of protons by nuclei. The section on Heavy Ion Dynamics and Related Processes reports on research into the $e^{+} e^{-}$problem and heavy ion dynamics. 
For each of the four main segments of this progress report, an overview presents the relevant philosophy and context of that segment of the research program. The progress reports addressing each specific topic can be located by the Table of Contents.

The Maryland Nuclear Theory program is broadly based with several separate and independently evolving collaborations among the members of the group and their colleagues from other institutions. The interests of the group are strongly directed towards fundamental questions of long-term interest.

The group atmosphere is open, informal, and highly interactive, in part due to the custom of daily discussions at the group lunch. A weekly seminar is held where invited visitors present recent research accomplishments. The seminar and visitor prograrns provide a rich atmosphere for postdoctoral associates and students. We try to encourage new research questions and new research methods, and to maintain an internal process of constructive criticism to help keep our standards high. We believe that the resulting program is vigorous and exciting, and that as a result, the quality of the graduate students, postdoctoral researchers, and visiting faculty who join our group is high, and their experiences with us are productive.

Our computing environment consists of a DECStation cluster with file service provided by a DECServer 3100 . A DECStation 5000 with $40 \mathrm{Mb}$ of fast memory has been added in the past year to provide compute service for large jobs. Three DECStation $3100^{\prime} s$ and seven DECStation $2100^{\prime}$ s ro'nd out the cluster. We have added $4 \mathrm{Mb}$ memory extensions to each of the 2100's in the past year. System management expertise has been provided by the University's Computer Science Center. A lorig-term arrangement for system support services is not yet in place but the Computer Science Center support is expected to fulfill the group's needs at this point.

The Nuclear Theory Group consists of six regular faculty members (Drs. M. K. Banerjee, T. D. Cohen, J. J. Griffin, W. M. MacDonald, E. F. Redish, and S. J. Wallace)

Promotion of Dr. Thomas Cohen to the rank of Associate Professor with tenure was approved by a very strong vote of the faculty in the fall of 1991 . The promotion is 
effective July 1, 1992. Professor Cohen holds a Presidential Young Investigator Award which commenced in the Fall of 1990. Dr. Wojciech Broniowski of the Institute of Nuclear Physics, Krakow, Poland, is spending twelve months at Maryland with support from Dr. Cohen's PYI funds. The entire group benefits from Dr. Broniowski's visit.' Professor John Tjon of the Institute of Theoretical Physics at the University of Utrecht visited the Nuclear Theory Group in the Spring of 1992. Professor Tjon has been a frequent visitor; his collaboration with the members of the Nuclear Theory Group is valued highly. We expect him to continue the association during the next contract year. Professor Brian Serot of Indiana University also visited the Nuclear Theory Group in May of 1992. Dr. Boris Ioffe of the Landau Institute, Moscow, visited Maryland for four weeks in April 1992 and spent part of his time with the Nuclear Theory Group and part of his time with the Elementary Particles Group. Dr. Georges Rupp from CFN/INIC, Lisbon, Portugal, spent a year the the Nuclear Theory Group from January, 1991 to January, 1992. Dr. Eugene Drukarev of the Leningrad Institute of Nuclear Physics, St. Petersburg, visited the Nuclear Theory Group for one week in May of 1992.

Dr. Antonio Delfino, Visiting Associate Professor from Universidade Federal Fluminense, Brazil, is here on sabbatical leave for two years (since March 1991) on a fellowship from the Conselho Nacional de Peqquisas-CNPq, Brazil.

Local faculty based at other institutions who are affiliated with the Nuclear : Theory Group include Dr. Maria Dworzecka of George Mason University. Close connections have continued with Dr. Hiroshi Ito of George Washington University. Two of our current postdoctoral research associates, Drs. Hilmar Forkel and Yuji Koike, will complete two-year appointments in the fall of 1992. Dr. Koike will leave to accept a position at Michigan State University. Dr. Forkel has accepted our offer of a one-year continuation of his postdoctoral position at Maryland. Dr. Derek Leinweber will stay with us for the second year of his two-year appointment. Two new postdoctoral associates will join the group in September 1992: Drs Joseph Milana from The College of William and Mary and Dr. Hung Jung Lu from Stanford Linear Accelerator Center. 
Current Ph.D. students include C. Edward Bell, Neal Devine, Edward Smith-Rowland, Xuemin Jin and Myunggyu Kim. Bell, Devine and Smith-Rowland are expected to complete their Ph.D. theses in 1992. 


\section{Hadrons in Nuclear Matter}

The question of how hadrons behave in the nuclear environment is one of the central questions in nuclear physics. Ultimately we believe that all strong interaction physics can be explained in terms of the quark and gluon degrees of freedom of QCD. On the other hand, decades of experience suggest that nuclei can be thought of in terms of interacting hadrons. One might imagine organizing the very complicated quark and gluon dynamics into two parts: the formation of hadrons and the many-body physics of interacting hadrons. The physics which we wish to explore is the possible feedback between the two pieces of this orgarization: when hadrons are in nuclei the many-body interactions may cause the internal structure of the hadrons to change and such changes can affect the many-body dynamics.

There are a large number of theoretical approaches to the problem of hadrons in the nuclear medium. The group at Maryland is exploring several of them. One extremely promising approach being pioneered at Maryland is the generalization of QCD sum rule techiques (which have proved to be a valuable nonperturbative technique for the study of hadrons in the vacuum) to problems at finite nuclear matter density. An early, highly simplified QCD sum rule treatment suggests that the strong scalar and vector nucleon self-energies of the Dirac phenomenology arise naturally from QCD. To go beyond this simple analysis, a variety of technical issues were confronted and a new calculational technology developed. Preliminary results based on the more sophisticated calculation supports our earlier qualitative work.

Another approach is to use models which attempt to simulate the relevant aspects of QCD in a tractable wy. Members of the group here have studied two different models of the nucleon. One set of studies is based on the color dielectric model in which the effects of finite nuclear matter density are implemented through the scalar and vector fields. The interaction of the valence quarks with these fields produces changes in the structure of the nucleon. Apart from the usual increase of the nucleon size, the study also produces density dependent coupling constants. The implications of these results on the 
properties of nuclear matter are under study. An alternative scheme, motivated by large- $N_{c}$ soliton models (such as the Skyrme model) is to simulate the effects of the medium (which localizes the soliton) by imposing hypersphere boundary conditions with some radius $R$ (where the density $\sim R^{-3}$ ).

We have also dealt with a number of other issues associated with hadrons in nuclei. For example we have shown how the coherent photoprouction of the $\pi_{0}$ serves as an ex serimental probe of the properties of vector mesons in the medium. We have also reviewed the question of whether there is convincing experimental evidence that the axial coupling of the nucleon, $g_{A}$, in the nuclear medium is greatly modified from its free space value. Finally, we have done some studies of hadronic matter at finite temperatures. Such studies may provide important insights for RHIC physics.

\section{A. QCD Sum Rules and Nucleons in Nuclear Matter}

This is an ongoing collaboration with Dick Furnstahl (of Ohio State), and David Griegel (of Indiana University), in which we are using QCD sum rule technology to explain the origins of the large Lorentz scalar and vector contributions to the nucleon optical potential in nuclear matter.

The QCD sum rule approach has been successful in describing properties of hadrons in free space. The approach is based on matching a QCD operator product expansion (OPE) description of a correlation function of currents carrying hadronic quantum numbers with a phenomenologial description. The nonperturbative QCD physics is expressed in terms of condensates-vacuum matrix elements of composite quark and gluon operators. The matching of the QCD and phenomenological descriptions is greatly aided by a Borel transformation which emphasizes the low dimensional (and thus tractable) terms in the OPE and the low mass states in the phenomenological description.

The basic idea, described in Griegel's thesis, was that the change in the scalar condensate in medium (which can be related to the $\sigma$ term in $\pi-\mathrm{N}$ scattering) leads to a large scalar attractive self-energy while the "vector condensate" (the density), leads to a 
large repulsive vector self-energy. We have put the theory on a much firmer theoretical foundation by

a) establishing the existence of a spectral representation relating a phenomenological description to a QCD description;

b) developing a transform analogous to the Borel transform for the free space case which emphasizes the contribution of the leading order terms in the operator product expansion and the low energy nucleon (as opposed to antinucleon) contributions;

c) calculating the Wilson coeflitients for all operators of dimension 5 or less;

d) demonstrating the insensitivity of our results to the choice of the Bcrel mass. The phenomenology appears to require that dimension 6 condensates in medium are similar to their free space values. Our earlier work on this subject has already had considerable impact.

(T. D. Cohen, X. Jin, R. J. Furnstahl [Ohio State], D. K. Griegel [Indiana])

\section{B. QCD Condensates in Nuclear matter}

To implement QCD sum rules, it is necessary to know the values of the condensates, i.e., the ground state expıctation values of composite operators constructed from the quark and gluon field operators. Our primary interest is in finding these condensates in the ground state of nuclear matter. There are many more condensates in nuclear matter than in the vacuum sin e many operators which vanish in the vacuum due to Lorentz invariance do not vanish in the nuclear medium-recall that nuclear matter breaks Lorentz invariance and is charasterized by a four vector $u_{\mu}$, the four velocity of nuclear matter.

We have estimated all of the condensates up to dimension five to first order in a density expansion-i.e., we assume that for some QCD operztor $\mathcal{O}$, the in medium condensate $\langle\mathcal{O}\rangle_{\rho}$ is simply $\langle\mathcal{O}\rangle_{\text {vac }}+\rho\left\langle N\left\|\int d^{3} x \mathcal{O}\right\| N\right\rangle$, i.e., a simple folding of the density with the nucleon matrix element. We have estimated the nucleon matrix elements using a combination of phenomenological inputs and modeling: 
- The scalar quark condensate is obtained from the $\sigma$ term extracted from $\pi-\mathrm{N}$ scatîering

- The scalar gluon condensate is estimated from the nucleon mass plus the QCD trace anomaly.

- Certain dimension four and five quark condensates can be related to nucleon structure functions obtained in deep inelastic scattering.

- Othe: dimension four and five quark operators can be related via a bag model to condensates extractable from deep inelastic scattering.

- Quark-gluon mixed condensates are estimated via bag models.

The model dependence of some of these condensates is rather large but fortunately the QCD sum-rules appear to be very insensitive to these condensates.

Finally, we have checked our low density assumption for the scalar quark condensate by building extremely crude models (which in fact is the best one can do). Fortunately, we have found that in all of the models the deviation from the low density approximation was small at nuclear matter density.

(T. D. Cohen, X. Jin, R. J. Furnstahl [Ohio State], D. K. Griegel [Indiana])

\section{Vector Mesons at Finite Temperature/Density from QCD Sum Rules}

There has been growing interest in the change of a hadron's properties at finite temperature $(T \neq 0)$ in connection with relativistic heavy ion collisions at SPS (CERN) and RHIC (BNL). In this work [1], we have developed a consistent and systematic formalism of finite temperature QCD sum rules for vector mesons, taking into account the following points: (1) At $T \neq 0$, there is no Lorentz invariance and thus nonscalar condensates such as $\left\langle\bar{q} \gamma_{\mu} D_{\nu} q\right\rangle_{T}$ survive. (2) At low temperature $\left(T \leq T_{c}\right)$, the whole $\mathrm{T}$-dependence can be properly incorporated in the condensates and not in Wilson 
coefficients. The critical observation to carry out the program is that the finite $T$ vacuum can be described as noninteracting Goldstone boson (pion) gas at low temperature. In this scheme, T-dependence appears through pion matrix elements of the operators appearing in OPE and the pion distribution function. Soft pion theorem gives reliable estimates for scalar condensates, and the pion matrix elements of nonscalar operators can be associated with pion structure functions measurable in hard processes (deep inelastic scattering, Drell-Yan, direct photon, and so on). We applied this method to $\rho, \omega$ and $A_{1}$ mesons and found that $m_{\rho, A_{1}}$ decreases at $T \neq 0$ (typically by $100 \mathrm{MeV}$ at $T=150 \mathrm{MeV}$ ) but $m_{\omega}$ does not change. We included the condensates up to dim. 6 . It turns out that $\mathrm{T}$-dependence of scalar condensates appear as an effect approximately proportional to $T^{2} e^{-m_{\pi} / T}$ and that of nonscalar condensates appears with factors of $T^{4}$ and $T^{6}$ instead of $T^{2}$. Therefore $T$-dependence of the vector meson masses are strongly suppressed at low $T$ $(T \leq 120 \mathrm{MeV})$. This is in contrast to the finite density case [2], where a linear decrease of $m_{\rho, \omega} \phi$ is observed. The effect of the pion structure functions is suppressed even more. We also found that the most important factors to determine $m_{\rho, A_{1}}$ are the four quark condensates, $\left\langle(\bar{q} \Gamma q)^{2}\right\rangle_{T}\left(\Gamma=\gamma_{\mu}, \gamma_{5} \gamma_{\mu}\right)$. One of the most important consequences is that $m_{\omega}(T)=m_{\omega}(0)$ since pion matrix elements of scalar operators appearing in OPE for the isoscalar correlator (for $\omega$ ) vanishes in the chiral limit. Therefore the splitting between $m_{\rho}$ and $m_{\omega}$ becomes bigger at $T \neq 0$. We note that our treatment of the $T \neq 0$ medium is valid only up to $T \leq 150 \mathrm{MeV}$, since at $T \geq 150 \mathrm{MeV}$ the effect of heavier resonances and their correlations become important.

As for the QCD sum rules in the nuclear matter, we can repeat the analysis above by replacing the pion by the nucleon. We need nucleon structure functions in this case and they are more available than for the pion. In the nuclear medium, the effect of the nucleon structure function is substantial, since they appear as an effect proportional to nucleon density. We found the linear decrease of vector meson mass for $\rho, \omega, \phi$ as a function of the density. This work is in progress for other vector mesons $\left(K^{*}, A_{1}\right.$, and $\left.J / \psi\right)[2]$. 
(Y. Koike, T. Hatsuda [INT], S. H. Lee [Yonsei Unversity, Korea])

[1] T. Hatsuda, Y. Koike and S.H. Lee, "Finite Temperature QCD Sum Rules Reexamined: $\rho, \omega$, and $A_{1}$ Mesons", UMD PP \#92-203.

[2] T. Hatsuda and S.H. Lee, INT-91-00-02 (1991); T. Hatsuda, Y. Koike and S.A. Lee, contribution to "Baryon '92", "QCD Sum Pules for Vector Mesons in Nuclear Medium".

\section{Nucleon in Nuclear Matter}

We use the Toy model of baryon bag formation to study the changes in the structure of a nucleon when it is placed in nuclear matter. This model is a derivative of the chiral confining model. The latter, develuped at Maryland, is based on the Nielsen-Patkos color dielectric model. The chiral contining model has, in principle, all mesons as dynamical variables. The Toy model is obtained by fixing the meson fields at their vacuum values.

In nuclear matter we use the values of the meson fields in nuclear mattier. Several interesting qualitative results have been found. We find that $M^{*}$, the nucleon mass in nuclear matter, does not scale with $F_{\pi}^{*}$, the latter being the value of the $\sigma$ field in nuclear matter. The product $M^{*}\left(r^{2}\right\rangle^{\frac{1}{2} *}$ is nearly independent of nuclear density. The coupling constant $g_{\sigma N N}^{*}$ decreases with density. The coupling constant $\frac{g_{j N N}^{*}}{M^{*}}$ and the rho-nucleon tensor coupling constant, $f_{\rho N N}^{*} / 2 M^{*}$, increase with density at the same rate. The coupling constants $g_{\omega}^{*}$ and $g_{\rho}^{*}$ also increase at the same rate, but the rate itself is low. We find that $M^{*}+g_{\sigma N N}^{*}\left(F_{\pi}-F_{\pi}^{*}\right)$ is approximately independent of nuclear density. The magnetic moments increase, while $g_{A}^{*}(0)$ decreases. It half the ncrmal density these changes are in the range of $3-7 \%$ depending on the parameters 'sed. We discuss briefly the implications of these results on the properties of nuclear matter.

(M. K. Banerjee, Phys. Rev. C45, 1359 (1992).) 


\section{E. A New Approach to Dense Matter in the Nambu-Jona-Iasinio Model}

The baryon structure and its modifications in nuclear matter become increasingly important at higher densities and are essential for the understanding of the expected deconfinement and chiral restoration phase transitions. This led recently to the investigation of nuclear matter properties in the context of various QCD-inspired baryon models. The extended baryon structure, however, restricts their application presently to a unit-cell approach, familiar from solid state physics. The nuclear matter is divided into identical cells, containing one unit of baryon number each.

The basic idea of the hypersphere approach is to effectively compactify these cells to a three-dimensional sphere, thus modeling part of the interactions with the dense environment by space curvature. This approach reproduces the results of skyrmion array calculations and, in particular, the chiral restoration phase transition at high densities almost quantitatively. In order to understand the underlying mechanism and the chiral phase transition explicitly in terms of quarks, we investigate the Nambu-Jona-Lasinio (NJL) model in the same framework. In contrast to the Skyrme model it is not a soliton model, it is based solely on quark fields (which leads to conceptually novel features on the hypersphere), and chiral symmetry breaking occurs dynamically.

A high-density phase transition to chiral restoration emerges and the density dependences of quark condensate and dynamical quark mass are very similar to those obtained in the conventional chemical-potential approach. In addition, the new approach allows the study of baryon-density free cavities in dense matter. These investigations are currently extended to the study of meson properties in dense matter. Their generalization to finite temperature is also in progress.

[1] H. Forkel, Dynamical Chiral Resioration in Dense Matter in Hyperspherical Unit Cells, Phys. Lett. B (1992), to appear.

[2] H. Forkel, A New Approach to Finite Baryon Density in the Nambu-Jona-Lasinio Moclel, University of Maryland preprint \#92-085, (1991). 


\section{F. Passage of High-Energy Partons through a Quark-Gluon Plasma}

Energy loss of high $p_{T}$ particles might signal the formation of the quark-gluon plasma (QGP) in relativistic heavy ion collisions (RHIC). With this in mind we estimated the stopping power of QGP acting on a single colored parton ( a quark or a gluon) and a color-dipole ( $\sim$ a heavy quarkonium) traversing a QGP. We calculated ihe energy loss of these external probes due to the elastic collision with plasma constituents taking into account the Debye screening effect in the framework of semiclassical kinetic theory. In this method, one gets the expression for the color dielectric tensor which is the same as the gauge invariant part of the one rbtained by perturbative QCD at finite temperature. We considered the QGP with massless constituents an at zero chemical potential which might be formed at the central rapidity region of RHIC. The results obtained can be summarized as follows:

1. The stopping power (energy loss per unit length) is proportinal to $\alpha_{s} m_{D}^{2}$ where $\alpha_{s}$ is the strong coupling constant and $m_{D}$ is the Debye mass. For a typical jet energy $(\sim$ $10 \mathrm{GeV}$ ), the stopping power for a single colored parton turns out to be $0.4 \sim 1.0$ $\mathrm{GeV} / \mathrm{fm}$ depending on the above plasma parameters. This is comparable to the string tension $(\sim 1 \mathrm{GeV} / \mathrm{fm})$ which is expected to be the typical magnitude of stopping power in the confined phase.

2. The energy loss of a color-singlet dipole due to the "long range" effect (momentum transfer smaller than the inverse size of the dipole) is smaller than that for a colored parton about one order of magnitude because of the color screening (color transparency). An extremely high-energy dipole whose energy is larger than its inverse transverse size, however, can lose energy by short distance collisions. In this case, the stopping power becomes the incoherent sum of each parton's stopping power, although it generally disrupts the coherent motion of the pair. 
At high energy, the energy loss due to radiation (bremsstrahlung) is expected to become important. It is also important to estimate the effect of non-equilibrium of the plasma. Work on these issues is in progress.

(Y. Koike and T. Matsui [Indiana University])

(Y. Koike and T. Matsui, Phys. Rev. D45, 3237 (1992).)

\section{G. Coherent Photoproduction of the Neutral Pion as a Test of In-Medium Vector Meson Masses}

In this work, we have calculated the coherent pholoproduction cross section on heavy nuclei and have shown that it is extremely sensitive to the effective mass of the vector meson in nuclear matter. The physics is bas ad on an analogy to the Primakoff process. In our case, the photon scatters off the coherent $\omega$ meson field field induced by the baryon density and this produces a photon from the $\pi-\gamma-\omega$ vertex. This process is dominant in the very forward direction. The strength of the interaction depends on the properties of the $\omega$ meson in the medium. Although the absolute magnitude of the cross-section depends on uncertain phenomenological inputs (in particular $g_{\omega}$ ) the shape of the differential cross-section, and in particular the position of the peak, are far more sensitive to the effective $\omega$ mass. This should provide a good experimental test of Gerry Brown's hypothesis that effective hadron masses decrease in the nuclear medium.

(T. D. Cohen and E. K. Smith-Rowland)

\section{H. The Axial Coupling in Nuclear Matter}

We have restudied the classic evidence of Buck and Perez that the axial coupling, $g_{A}$, is quenched in nuclei to approximately 1 . This approach involves a correlation of Gamow-Teller strengths to magnetic moments. We found that meson exchange current (MEC) effects were essentially neglected in this approach. The inclusion of MEC dramatically alters the results and suggests that there is no state independent quenching. 
(T. D. Cohen and L. S. Kisslinger [Carnegie Mellon University])

\section{The Structure of Hadrons}

The problem of hadronic structure remains one of the most interesting and vibrant problems in strong interaction physics today. It is virtually universally agreed that ultimately, hadrons can be described in terms of QCD, the fundamental theory of the strong interactions. The central difficulty, of course, is that the strong interaction at low momentum transfers is, in fact, strong and hence perturbation theory is not viable. There are a number of major approaches to this problem which are being explored at Maryland.

One approach, developed in the late 1970's and early 1980's primarily in the Soviet Union, is the so-called QCD sum-rule method. In this approach, one uses an operator product expansion along with phenomenological condensates to calculate correlation functions valid in the deep Euclidean region. The idea is to use spectral representations to match these to phenomenological descriptions of the spectrum. Various mathematical transforms are used to improve the matching. Whether or not the matching works for a particular channel depends in large measure on delicate details and because of this the amount of information extractable is rather limited. Nevertheless this is a powerful technique. Our main interest in using this techique has been for propagation of hadrons in nuclear matter. However, we have also worked on calculating the so-called $\sigma$ term of $\pi-\mathrm{N}$ scattering. This is an interesting property of the nucleon in its own right and is an important phenomenological input to our in-medium sum rule studies.

From an intellectual point of view, perhaps the most straightforward approach to hadron siructure from QCD is via computer simulations on the lattice. One "simply" evaluates the functional integral for correlation functions in a lattice regularized version of the theory using a Monte Carlo technique. The recent work at Maryland has centered on electro-magnetic properties of hadrons including transition moments and the properties of the decuplet baryons.

It should be recalled of course that lattice calculations have a number of extreme 
limitations. For technical computational reasons, the lattice spacing is too large to resolve fine details of the structure and, perhaps more seriously, it is probably too large to be completely in the scaling regime (which is necessary for an extrapolation to the continuum). Moreover the lattices used are typically somewhat small (i.e., not much larger than hadronic sizes) and hence finite volume effects may be important. Moreover, there are typically fairly large statistical errors which prevent one from obtaining much detailed information. Finally, the quark masses used are typically much too large which may greatly change the physics-particularly if pions are important. It is clear that fully realistic and detailed lattice results are quite some time off.

Thus, given the limitations of both QCD sum rules and lattice calculations, it is iniportant as a practical matter to develop models of QCD which reproduce the essential QCD physics for particular phenomena in a tractable form. Models will remain of great theoretical importance even when reliable lattice calculation become avaliable. Computer results give answers without explanations. Models are invaluable for providing insights into the essential physics of the problems. The key to model building is the identification of the essential degrees of freedom. We are exploring a wide variety of models.

One class of model we have been studying for some time is the chiral confining model, a mean field treatment which generates confinement through the vanishing of the color dielectric function far from the soliton. The principal improvement made recently was the inclusion of an ' $t$ Hooft type interaction which simulates the role of instantons. This has produced remarkable imrovement in the phenomenology.

A second class of model being studied is a relativistic generalization of the conventional (Isgur type) quark model. Given the fact that excitation eneigies of hadrons are substantial fractions of their masses it seems a prori questionable to u se nonrelativstic models. One might hope to be ab'e to do relativstic quark physics witho' 1 t all of the complications associated with field theoretic treatments. To develop a filly relativistic finite particle number (i.e., non-field-theoretic) quark model one needs many-particle generalizations of the Dirac equations. The key to developing such many-body Dirac 
equations is the elimination of some standard pathologies which arise in naive treatments such as continuum dissolution, poor clustering properties or the asymmetric treatment of different particles. A quasipotential formalism for treating many Dirac particles which is free of these pathologies was developed here in the past few years and is currently being applied to the problem of hadron spectroscopy.

A third class of models we are considering are the so-called hedgehog models (which include the Skyrme model, the hybrid or chiral bag model and the chiral quark-meson model). In these models, the nucleon and delta are projected from the same intrinsic state in much the same way as states in a rotational band of a deformed nucleus are projected from an intrinsic Hartree-Fock state. Much of our recent interest in these models has centered on calculations of the proton and neutron electromagnetic polarizabilites (which have recently been measured with far greater accuracy than in the past.) The study of polarizabilities has proven to be very interesting both in its own right and also because it has provided a window into the structure of the hedgehog models. We have obtained important insights concerning the role of the $\Delta$ resonance and the connection of hedgehog models to chiral perturbation theory.

The general question of the relationship of chiral perturbation theory $(\chi \mathrm{PT})$ to various approaches to $\mathrm{QCD}$ has been an important recent concern of ours. $\chi \mathrm{PT}$ is based on the seperation of the scale of the pion mass from all other scales in hadronic physics. This allows a systematic expansion treating the pion mass squared (or alternatively the quark mass) as an expansion parameter. One nontrivial consequence of this approach is nonanalytic behavior in $m_{\pi}^{2}$ due to the infrared behavior of loops containing pions. We have investigated the connection of $\chi \mathrm{PT}$ to quark mass extrapolation of quantities calculated on the lattice, to the presence of pion cloud physics in the quenched approximation of QCD and to hedgehog models. The general question of the role of intermediate states which include the $\Delta$ resonance in $\chi \mathrm{PT}$ treatment of the nucleon has been studied in the context of the hedgehog models. 


\section{A. QCD Sum Rules and The Sigma Term}

The sigma term is a measure of the explicit chiral symmetry breaking term in the nucleon and it gives insight into how much the chiral condensate is altered inside a proton. The value of this parameter (which can be extracted experimentally in $\pi \cdot \mathrm{N}$ scattrering) is an important input to our nuclear matter calculations. We are in the process of using the techniques of QCD sum-rules to calculate this quantity. The key "trick" is to use the Feynman-Hellmann theorem. The sum-rule calculation presumably misses the physics of the pion cloud. Thus possible corrections due to chiral perturbation effects are also being explored.

(T. D. Cohen, R. J. Furnstahl [Ohio State] and D. Griegel [Indiana])

\section{B. Decuplet Baryon Electromagnetic Structure on the Lattice}

Through studies of hadronic electromagnetic form factors, the lattice gauge approach to QCD has proved to be a valuable tool in revealing the underlying quark substructure of hadrons. We have developed a fully relativistic formalism for isolating and extracting the four electromagnetic multipole form factors of spin-3/2 systems in lattice field theory. Results of the first lattice $Q C D$ analysis of $S U$ (3)-flavor decuplet baryons have been systematically examined to reveal new aspects of the underlying nonperturbative quark-gluon dynamics.

The lattice results show evidence of a spin-dependent force with a behavior analogous to what one expects from the hyperfine interaction term of the one-gluon-exchange potential. In contrast to our octet baryon analysis this spin-dependent force counteracts center-of-mass shifts and suppresses any baryon dependence of the quark electromagnetic properties. As a result, decuplet baryon electromagnetic structure is, for the most part, consistent with nondynamical models such as the simplest quark model based on $S U(6)$-spin-flavor symmetry broken only by the quark masses.

The $E 2$ form factor allows a preliminary glimpse of the shape of the decuplet 
baryon ground state. In our analysis, the $E 2$ form factor is at the threshold of confirming or rejecting the hedgehog Skyrmion description of baryons. A future high statistics lattice calculation on a cubic lattice will provide considerable insight.

(D. Leinweber)

\section{The $N \gamma \rightarrow \Delta$ Electromagnetic Transition in Lattice QCD}

Our current focus is on a lattice QCD investigation of the $N \gamma \rightarrow \Delta$ electromagnetic transition where there have been significant experimental efforts to measure the $M 1$ and $E 2$ transition moments. This, of course, has been accompanied by a plethora of model calculations for these quantities. The lattice results will be instrumental in assessing the reliability of the model analyses. We have made significant progress in this analysis.

(D. Leinweber)

\section{Effect of Instantons in the Chiral Confining Model}

Instantons are objects in the Euclidean world, so their role in a phenomenological model requires some explanation.

Instantons are classical solutions to the Euler-Lagrange equations of Euclidean QCD in the pure gluon sector. Thus they are expected to play an important role in the calculation of the QCD partition function. Integrating out the quark fields for a fixed gluon field configuration produces, in the partition function, the factor $\operatorname{det}(\not D)$, where $\not D=\gamma_{\mu}\left(\partial_{\mu}-i g A_{\mu}\right)$. When the gluon field is an instanton, the operator $\mathbb{D}$ has a zero eigenvalue, which, of course, makes $\operatorname{det}(\not h)=0$. If the quark sees any interaction, such as that with the quark condensate, the eigenvalue of $\operatorname{det}(\not D)$ is shifted to a nonzero value and, what is more crucial, the shift plays an important role in determining the value of the partition function. Thus the instantons have to be taken account of even in the quark sector. 
' $t$ Hooft found that the effect of the instantons on the quark determinant can be approximately duplicated by adding a $2 n_{f}$-fermion interaction term in the lagrangian of the form

$$
\rho^{-3} \operatorname{det}\left|2 \pi^{2} \rho^{3} \bar{\psi}_{\bullet}\left(1+\gamma_{5}\right) \psi_{t}\right|+h . a .+\cdots,
$$

where $\rho$ is the 'size' of the instanton and $s, t$ are flavor labels. This $n_{f} \times n_{f}$ determinantal interaction is known as the ' $t$ Hooft interaction.

The " $t$ Hooft interaction is easily translated into the Minkowski form and, thus, can be used in phenomenological models. The ' $t$ Hooft interaction is invariant under chiral $S U_{L}\left(n_{f}\right) \otimes S U_{R}\left(n_{f}\right)$. But it breaks chiral $U(1)$ invariance. ' $t$ Hooft himself pointed out how it can help solve the farnous $\eta^{\prime}$ mass problem.

There is an impressive body of literature using the ' $t$ Hooft interaction either directly in a Nambu Jona-Lasinio model or after 'translating' it into a mesonic form.

Our chiral confining model has the major flaw that it gives for the dimensionless quantity $M_{N}\left\langle r^{2}\right\rangle^{\frac{1}{2}}$ a value $\sim 7$, which is nearly twice the experimental value of 3.85 . Almost nothing appears to reduce it. We clearly need an attractive short-ranged force. The ' $t$ Hooft interaction is precisely of this form.

However, our model is based on the Nielsen Patkos model. The latter introduces a generalized block spinning to obtain a coarse-grained gluon field and the associated notion of a color dielectric function. In order for us to use the " $t$ Hooft interaction in our model we must demonstrate that the Nielsen-Patkos model has instantons. We have shown that this is indeed the case. Once the instantons are shown to exist, the rest follows and one has the 't Hooft interaction for the Nielsen-Patkos lagrangian.

We have argued before that it is proper to use quark fields simultaneously with meson fields with the provision that no quark loops should be included. The effects of the latter are taken care of by the mesonic lagrangian. The quarks can be eliminated completely in favor of mesons if the valence quark eigenvalue in the background meson fields is negative. It appears not to happen in any confining model. Thus we must retain the ' $t$ Hooft interaction, for treatment at the tree level, while including its axial $U(1)$ 
breaking effects in the mesonic lagrangian. The axial $U(1)$ breaking terms in the mesonic lagrangian have negligible effect on the nucleon structure problem.

We use the 't Hooft interaction for two flavors, up and down, for which it is an attractive $\delta$-function quark-quark interaction. The implied ultraviolet divergence is not present in the original form. One way of regulating the divergence in a translationally invariant way is to use a momentum cut-off. This is cumbersome for a mean field calculation. The comparison of the exact form of $\operatorname{det}(\mathbb{D})$ with the result from 't Hooft interactions suggests a natural regulating factor, $\left(\frac{r^{2}}{r^{2}+\rho^{2}}\right)^{6}$, which we use.

The ' $t$ Hooft interaction, as a two-body force, contains the factor $1+\vec{\tau}_{1} \cdot \vec{\tau}_{2}$, which, within a factor of $\frac{1}{4}$, is a projector of the isosinglet state. Since, typically $\rho \sim 0.25 \mathrm{fm}$, the quarks will interact dominantly in space symmetric states. Thus the interaction is effective only in singlet-singlet states of two quarks. It provides attraction in the nucleon state and has zero effect in $\Delta$, since the latter has only triplet-triplet pairs.

The " $t$ Hooft interaction is too strong to be treated perturbatively. At the minimum one must do a new mean field calculation including it. Its strong spin and isospin dependence can be handled using the hedgehog ansatz.

We have done such calculations and have obtained preliminary results. The table below shows some typical results for the Toy model, with and without instantons $(\rho=0.27 f m)$.

\begin{tabular}{||l|c|c||}
\hline \hline Quantity & $\begin{array}{c}\text { without } \\
\text { Instanton }\end{array}$ & $\begin{array}{c}\text { with } \\
\text { Instanton }\end{array}$ \\
\hline Mass $(\mathrm{MeV})$ & 1637 & 1323 \\
\hline$\left\langle r^{2}\right\rangle^{\frac{1}{2}}(\mathrm{fm})$ & 0.95 & 0.85 \\
\hline$M a s s \times\left\langle r^{2}\right\rangle^{\frac{2}{2}}$ & 7.9 & 5.7 \\
\hline$M_{\Delta}-M_{N}(\mathrm{MeV})$ & 0 & 331 \\
\hline$\mu_{p}(\mathrm{nbm})$ & 3.44 & -2.29 \\
\hline$\mu_{n}(\mathrm{nbm})$ & 2.62 & -1.75 \\
\hline$g_{\pi N N} \frac{m_{\pi}}{2 M}$ & 1.03 & 0.98 \\
\hline \hline
\end{tabular}


For large number of colors, $N_{c}$, the instanton contribution to $N$ and $\Delta$ goes as $\frac{N_{u} N_{d}}{N_{c}^{2}}$ where $N_{u}$ and $N_{d}$ are the numbers of up and down quarks in the multiplet of highest charge ( $\mathrm{p}$ and $\Delta^{++}$). It is easy to see that $M_{\Delta}-M_{N}$ goes as $\frac{1}{N_{c}^{2}}$ for large $N_{c}$. It is also easy to see how inappropriate it would be to ignore the role of instantons on the basis of $1 / N_{c}$ expansion.

We feel that inclusion of the instanton effect through the ' $t$ Hooft interaction has brought the chiral confining model to a satisfactory stage. The value 5.7 for Mass $\times\left\langle r^{2}\right\rangle^{\frac{1}{2}}$ will be brought down further through (i) corrections for center of mass motion to both factors and (ii) inclusion of quark-quark correlations to allow for two quarks in singlet-singlet state to be closer together.

(M. Kim and M. K. Banerjee)

\section{E. Relativistic Quark Model of $\bar{q} q$ Mesons}

The two-body equation

$$
\left[\left(E_{1}-h_{1}\right) \frac{h_{2}}{\epsilon_{2}}+\left(E_{2}-h_{2}\right) \frac{h_{1}}{\epsilon_{1}}-V\right] \Psi=0
$$

where $h_{i}=\alpha_{i} \cdot p_{i}+\beta_{i} m_{i}$, and $\epsilon_{i}=\sqrt{p_{i}^{2}+m_{i}^{2}}$, which was developed by Mandelzweig and Wallace, is being used to describe the bound states of quark-antiquark mesons. We have developed a computer program to solve the two-body equation in momentum-space using an expansion of the wave function in cubic splines as suggested by Spence and Vary. We have reproduced the existing results for heavy quark states ( charmonium and bottomion mesons) using a conventional potential model ansatz of a linear confining potential (scalar) plus a Coslombic gluon-exchange interaction (vector). Lattice gauge calculations, using the quenched approximation, provide a justification for the linear potential with heavy quarks. We use the same potential for light quarks.

Solutions of the problem in various limiting cases have been obtained numerically to test the methods used to handle the singularities of the potential at $q=0$. In the non-relativistic limit our momentum-space code, using just the linear potential, yields 
bound state energies and wave functions in very good agreement with known analytical results. Also the two-body equation yields good agreement with a relativistic virial theorem that relates the kinetic and potential energies. We have subsequently been applying it to lower mass quarks to study the large mass splitting of $0^{-}$and $1^{-}$states $(\pi$ and $\rho$ mesons).

It is known that substantial $\pi-\rho$ splitting can be obtained using the $\alpha \gamma_{1} \cdot \gamma_{2} / \mathrm{r}$ term in relativistic equations. However the equations used to show the splitting do not have a good co.nection to field theory while the two-body equation above does. With $\alpha / \mathrm{r}$ type potentials there arises a singularity as $\mathrm{r} \rightarrow 0$ and this can lead to a problem with the normalizability of the wave function. Tiemeijer and Tjon have performed an $r$-space analysis of a quasipotential equation identical to the two-body equation above and they showed that there is a critical coupling $\alpha_{\text {critical }}$ above which normalizable solutions do not exist. We found that retardation corrections to the $\alpha / \mathrm{r}$ potential greatly suppress couplings of ++ states to -- states, and similar suppressions holds for couplings of -to,+++- to -+ and -+ to +- states. The momentum space analysis that we use can include the retardations in the various couplings and thus achieve more physically acceptable results. We still obtain the critical behavior.

Furthermore Tiemeijer and Tjon have indicated that reasonably good values for the $\pi$ - $\rho$ mass difference could be obtained. When we employed their potential, which was of the form $\kappa \mathrm{r}+\alpha \boldsymbol{\gamma}_{2} \cdot \gamma_{2} / \mathrm{r}+\mathrm{C}$, we were able to obtain good agreement with their results for $\alpha<\alpha_{\text {crst }}$. Since the two body equation is charge congugate invariant, there is a negative-energy solution corresponding to each positive-energy one. The interesting feature of the solutions is that the mass gap between positive- and negative-energy solutions goes to zero very quickly as $\alpha \rightarrow \alpha_{\text {critica! }}$. This sudden collapse of the mass gap will be referred to as diving. The mass lowest positive-energy solution is plotted in the diagram. The corresponding negative-energy solution is not shown but it is a perfect reflection of the plot with respect to the horizontal axis.

The value of $\alpha_{\text {critical }}$ is about $10 \%$ larger in our numerical solution than in 


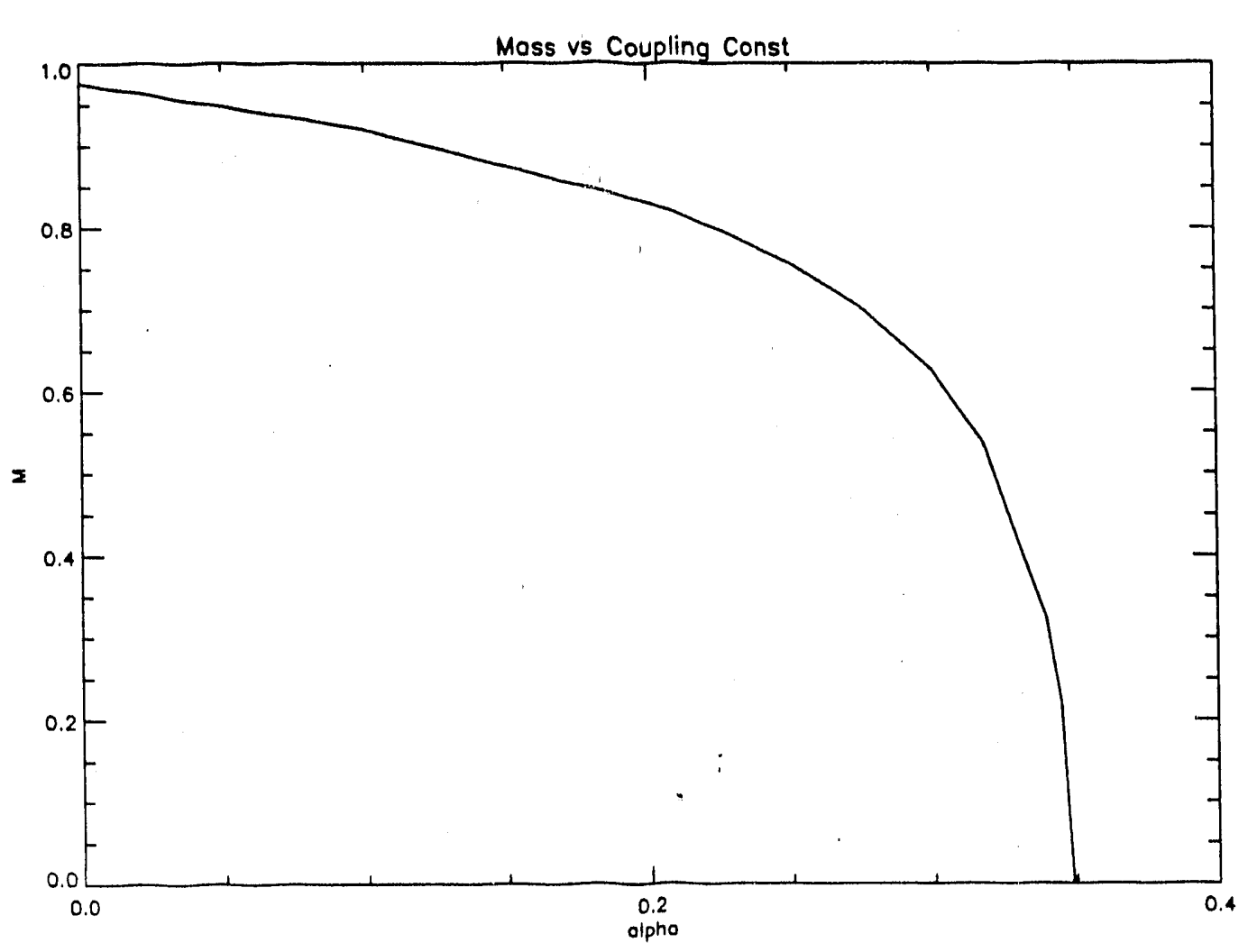

Fig. $1 \mathrm{~A}$ plot of the mass of the $0^{-}$bound state (in $\mathrm{GeV}$ ) vs the gluon-exchange coupling constant, $\alpha$. Here the quark mass $M_{q}=.250 \mathrm{GeV}$, $\mathrm{C}=-.529 \mathrm{GeV}$ and $\kappa=0.2 \mathrm{GeV}^{2}$

Tiemeijer and Tjon's analytical result. We have found that $\alpha_{\text {critical }}$ value is altered when retardations are included as can only be done in our momentum-space analysis. An interesting question is how the vanishing of the mass gap varies with the quark mass. Vanishing of the mass gap for small quark masses is expected in that QCD should have a spontaneous breakdown of the perturbative vacuum by pair formatiom. Since the diving is rather insensitive to the confining potential, the same phenomenon is expected to take place in QCD as in the model.

Currently we are working on how the retardation changes the precise value of $\alpha_{\text {critical }}$ and in what way retardations affect the diving behavior of the bound state solutions for small quark masses. Also we are examining if there is a similar kind of diving behavior for the bound state as we lower the quark mass, i.e., whether there is a critical quark mass, $m_{\text {critical }}$, where the mass gap between positive and negative energy states goes to zero for a fixed value of $\alpha$. Preliminary results do indicate the mass gap 
decreases as the quark mass is lowered. Since the effect depends on the short distance behavior of one-gluon exchange, we also will incorporate the running of the coupling constant which softens the singularity at $r=0$.

(C. E. Bell (Ph.D. thesis) and S. J. Wallace)

\section{F. Nucleon Electromagnetic Polarizibilities in Chiral Models.}

The electromagnetic polarizabilities of the nucleon have recently been extracted from precise Compton scattering experiments. We are calculating the polarizabilities in the context of models which reproduce both the chiral and large $N_{c}$ behavior of $\mathrm{QCD}\left(N_{c}\right.$ is the numbei of colors in QCD). Such models have been widely popular during the past decade (in part due to Witten's flirtation with the Skyrme model) and it is interesting to see whether they can explain the data. In these models the nucleon is the projection from a "hedgehog intrinsic state". Our calculation was based on a semiclassical treatment of the model, using the standard "cranking"-like projection scheme developed here and using linear response theory. The organizing principal for this calculation is the $1 / N_{c}$ expansion. We have shown that the electric polarizability is of order $N_{c}$ while the difference of the proton and neutron polarizabilites is order $1 / N_{c}$. The paramagnetic part of magnetic polarizability is order $N_{c}^{3}$ due to the near degeneracy of the $\mathrm{N}$ and $\Delta$ and the fact that the $N$ and $\Delta$ have the same intrinsic state leading to an $N-\Delta$ transition magnetic moment of order the nucleon magnetic, moment (i.e $\mathcal{O}\left(N_{c}\right)$.

We encountered and solved a surprising number of technical issues in this apparently simple problem. One key issue was the treatment of zero modes in projection and the guarantee that the Thompson limit is reproduced. Another key issue is the relationship with chiral perturbation theory which is rather subtle since the large $N_{c}$ and chiral limits do not commute We found that all previous work on the subject was deeply flawed.

(T. D. Cohen, W. B. Broniowski [Cracow and Maryland], and M. K. Banerjee) 


\section{G. Chiral Corrections to Lattice Calculations of Charge Radii}

Wattice gauge theory calculations of hadronic quantities must, for computational reasons, be done at unrealistically large quark masses and extrapolated down to the physical region. We have shown that in lattice calculations of the proton and pion electric charge radii, such extrapolations will give large systematic errors unless one includes terms nonanalytic in the quark mass in the extrapolation. These nonanalytic terms are calculable in chiral perturbation theory. We find effects as large as $50 \%$ in the mean square charge radius.

(T. D. Cohen and D. B. Leinweber)

\section{H. Are There Pion Loops In Quenched QCD?}

Lattice QCD calculations are often done in the so-called quenched approximation to greatly simplify the numerical calculation. This approximation involves setting the fermion functional determinant to zero in the functional integral and is equivalent to neglecting all quark loops which are not connected to external currents. Naively, one would think the physics of the pion cloud (i.e. chiral loops) is absent in this approximation since the pion includes quark-antiquark states. We have shown that this is not true. The quark propagators are fully relativistic and the "z-graphs" enable quark-antiquark states to be produced. A graphical analysis of the nucleon indicates that these z-graphs can create states with pionic quantum numbers. Moreover, a large $\mathrm{N}_{c}$ analysis shows that pion loop physics is leading order in $1 / N_{c}$ and at large $N_{c}$ is known to be quenched. In addition models such as the Skyrmion which correctly incorporate the large $N_{c}$ physics reproduce the chiral nonanalytic behavior associated with pion loops. This demonstration that pion loop physics is included in the quenched approximation is very important in attempts to interpret the lattice results.

(T. D. Cohen and D. B. Leinweber) 


\section{Hedgehog models and Chiral Perturbation Theory}

Hedgehog models are classical soliton models of the baryons in which the nucleon and delta are projected from the same intrinsic "hedgehog" state. These models include, for example, the Skyrme model. One feature of these models is that the pions in the models are classical. Another approach to hadron structure is "chiral perturbation theory" developed by Weinberg and others in which quantum pion loops can lead to nonanalytic behavior of various quantities in terms of $m_{\pi}^{2}$. We show that these nonanalytic terms are in fact present in the classical soliton models-that is, the quantum pion loop physics is simulated at the classical level. We also note that there is a persistent factor of three discrepancy between the coefficients of these nonanalytic terms in the hedgehog models compared to the chiral perturbation theory predictions. This discrepancy is because the large $\mathrm{N}_{\mathrm{c}}$ and chiral limits do not commute: In the chiral limit the delta-nucleon mass splitting is assumed to be much larger than the pion mass while, in the large $\mathrm{N}_{\mathrm{c}}$ limit, the delta-nucleon mass splitting is assumed to be much smaller than the pion mass. (In nature the two are of comparable scale). If chiral perturbation theory is redone including a degenerate $\Delta$ then the results agree with the hedgehog models.

(T. D. Cohen and W. B. Broniowski [Cracow and Maryland])

\section{Relativistic Nuclear Physics}

Many aspects of nuclear physics are explainable using conventional degrees of freedom, namely, mesons and nucleons. Although there does not exist a satisfactory or appropriate linkage to QCD, a sophisticated phenomenology based on mesons and baryons does give a good account of the $N N$ interaction and experimental data obtained with the current generation of electron and hadron accelerators used for nuclear experiments. At sufficiently high energy, there is convincing evidence that the underlying quark and gluon degrees of freedom are essential. A main task of research is to discover the linkage of the two pictures so as to put theoretical analyses on a sound footing. 
One possibilty is that electromagnetic probes of few-nucleon systems will provide the best tests of our fundamental understanding. The testing ground is clearly at fairly high momenta as mesonic models seem to work up to $q=1 \mathrm{GeV} / \mathrm{c}$. For such momenta, it becomes essential to include relativistic effects in a consistent way and for this reason, our work has emphasized relativistic dynamics for some years. One example is th: use of relativistic quasipotential equations to describe few-nucleon systems. Thesis student Neal Devine is nearing completion of an analysis of deuterium using the two-body Dirac equation developed by Mandelzweig and Wallace. Meson-exchange currents have been included and calculations of electromagnetic form factors have been done for large momentum transfer. Current conservation holds for elastic scattering in the quasipotential approach.

Our work on the relativistic analysis of the $N N$ interaction and the consequent predictions for proton-nucleus scattering has reached a new phase. Previous work has been related to elastic scattering based on optical potentials which were determined by a complete set of Lorentz-invariant $N N$ amplitudes and the nuclear density. This is referred to as the IA2 formalism. Inelastic scattering analyses have not been done in the IA2 formalism because of the massive effort required to develop new computer codes. However this problem can be finessed to a great extent by developing an effective interaction which incorporates the effects of the complete set of $N N$ amplitudes. An IA2 effective interaction can be calculated as a function of the nuclear density and it may be used in existing computer codes to perform interesting new calculations. Significant experimental efforts exist to study the effective interaction in p-nucleus scattering at Indiana and Los Alamos. Moreover there are indications from the work of Kelly and collaborators that the effective interaction should be close to the one which is obtained from the IA2 formalism. Therefore we are developing the IA2 effective interaction with full account of its spin and isospin dependence and we expect a fruitful interplay of theory and experiment for inelastic scattering. Most of the spin and isospin dependent terms in the effective interaction are not tested at all in elastic scattering and thus many new theoretical predictions become possible. 
An important aspect of the elastic scattering of protons is the full-folding treatment of the optical potential. The only calculations so far which have done the complete job are by a group at the University of Georgia using a nonrelativistic formalism. We have tried to understand the effects of full folding in light of our own results which show that off-shell effects in the optical potential are of minor importance. Our emphasis has been on performing the loop integration with a varying energy in the $\mathrm{t}$-matrix. However this has not yielded important effects and we are somewhat at a loss to corroborate the interesting effects found by the Georgia group. Therefore we have undertaken a momentum-space analysis with full folding using the complete off-shell t-matrix. This work will clarify the importance of full folding.

For some time we have been interested in few-body problems for Dirac particles. Currently calculations are being performed using the two-body Dirac equation with meson-exchange interactions to determine electromagnetic response functions of deuterium at high $q$. An outline of the extension of this formalism to three Dirac particles has been published in a conference proceeding, however, little work has been done on solving the three-body equations. Several complications arise such as the fact that the relativistic form of the kinetic energy is not diagonalized by use of Jacobi momenta. Indeed, partial wave analysis of the three-body equations is formidable. Therefore we are interested in direct numerical approaches which do not require partial wave expansions. We have begun to solve the three-body equations for a simple case using a direct numerical method. Dr. Antonio Delfino, who is visiting from Brazil, has taken a keen interest in this project and a working computer code has been developed for a simple case. We plan to apply the method to the relativistic three-particle equations.

\section{A. Boost, recoil, and Wigner rotation effects on no-pair analyses of proton elastic scattering}

'The relativistic 'no-pair' potential for elastic proton scattering is shown to reduce to a conventional $t \rho$ form using optimal factorization of the optical potential and the 
impulse approximation. The nucleon-nucleon t-matrix is needed in the Breit frame and it may be obtained by boosting the NN CM frame t-matrix. It is shown that the general form of the boost involves a Møller factor, Wigner rotation operators acting on spins and a Lorentz boost of momentum arguments. The reduction to $t \rho$ form clarifies how the 'no-pair' analysis differs from conventional Watson or Kerman, McManus and Tualer analyses which usually omit Wigner rotation effects and use a galilean boost of momentum arguments of the $t$-matrix instead of a Lorentz boost. Our recent calculations in momentum space have found rather minor off-shell effects based on the 'no-pair' analysis. Much larger off-shell effects based on the KMT formalism have been reported by C. Elster and P. C. Tandy in Phys. Rev. C40, 881 (1989), and in Proceedings of Ohio State Workshop, B. C. Clark, R. J. Perry, J. P. Vary, eds. (World Scientific, Singapore, 1989), p. 512.

In this paper we perform KMT calculations using a Lorentz boost of the NN CM frame t-matrix. The KMT result so calculated is in essential agreement with the 'no-pair' result showing that off-shell effects are minor, as shown in the figure. In contrast we find that large off shell effects can be obtained when a galilean boost is used in combination with inaccurate Coulomb corrections. The large effects found in the earlier KMT calculations by others are an artifact of the approximations used, e.g., the galilean boost, rather than a sign of large 'off-shell effects due to the $N N \mathrm{t}$-matrix.

The figure shows a comparison of off-shell and on-shell KMT results based on the $t \rho$ potential using the Breit frame $\mathrm{t}$-matrix and including the recoil terms and $(A-1) / A$ factors. The solid lines show the off-shell results. Dashed a: : dotted lines show two forms of on-shell results which differ in their treatment of nucleon-exchange contributions. The dotted lines show results based on using Yukawa fits of on-shell NN Lorentz-invariant amplitudes such that the optical potential may be localized. This permits nucleon-exchange terms to be separated and treated in a careful fashion. The dashed curves show an on-shell calculation in which the NN amplitudes are calculated assuming that they depend only on the momentum transfer, $q$, as has been used in the older KMT 

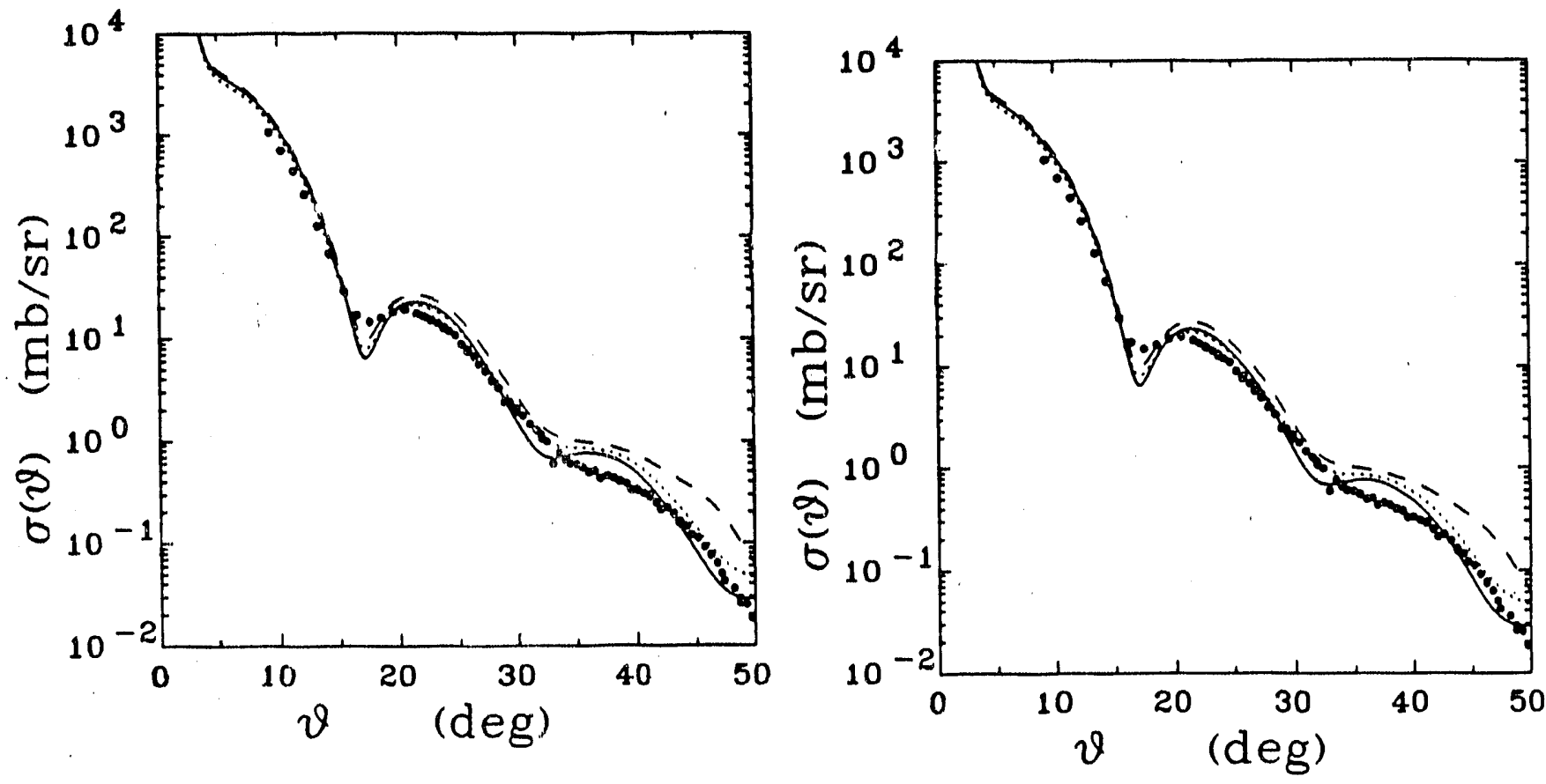

Cross section $(\sigma)$, polarization (P) and spin rotation function (Q) for $200 \mathrm{MeV}$ protons scattering from ${ }^{40} \mathrm{Ca}$. Solid lines show the KMT results based on off-shell

NN amplitudes. Dash lines show KMT results based on Yukawa fits of

Lorentz-invariant amplitudes. Dot lines show KMT results based on on-shell NN amplitudes using a cutoff such that amplitudes remain constant after reaching the maximum momentum transfer for on-shell scattering.

calculations.

The KMT calculation which includes Wigner rotations, a Lorentz boost of mornentum arguments of the t-matrix and accurate Coulomb corrections is in excellent agreement with our previous 'no-pair' analysis and the conclusion of minor off-shell effects.

(J. A. Tjon and S. J. Wallace, Phys. Rev C44, 1156 (1991).)

\section{B. Lectures on Relativistic Nuclear Physics}

A set of lectures delivered at the Dronten Summer School has been published in the past year. The lectures introduce students to new approaches to nuclear shysics which are based in part on relativistic quantum field theories of hadrons (mesons and 
nucleons) and in part on assumptioms regarding the connections of hadrons to underlying quark degrees of freedom.

Meson-exchange dynamics provides a very successful theoretical approach to nuclear physics. With some phenomenological vertex functions employed, one obtains an economical description of the $N N$ interaction. From the $N N$ interaction many nuclear phenomena can be understood such as the optical potential for nucleon-nucleus scattering and the mean fields which form the basis of the nuclear shell model. Moreover meson-exchange currents as needed to explain electromagnetic reaction are based on the same general framework.

Over the past seven years, the rellativistic approach has proven to be successful for describing proton-nucleus scattering observables. The development of complete sets of invariant amplitudes and the associated IA2 potential based on them provides a consistent unification of the relativistic $N N$ interaction and the Dirac optical potential. In a straightforward fashion one may interpret the success of the Dirac equation with large scalar and vector interactions as beirg due to implicit inclusion of virtual $N \bar{N}$ pairs. However recent work suggests another interpretation.

Analyses based on QCD sum rules applied to a nucleon in nuclear matter show that the decrease of the quark condensate from its vacuum value can be quite large in a nucleus. Such a decrease of the quark condensate generates large scalar and vector interactions similar to those found from the Lorentz-invariant analysis of $N N$ scattering. In this interpretation, virtual $N \bar{N}$ couplings would at the same time be suppressed. Thus it is possible that the virtual pair interpretation is unnecessary. According to this interpretation the relativistic framework allows for distortion of the positive-energy Dirac spinors which go into defining the effective interaction as defined above. One need not assign the same physical meaning to the admixing of positive and negative-energy states of the particle as is usually done for point particles, e.g., in QED. In any event, the distortion of the Dirac plane waves is the essential ingredient and it. allows a simple extensicn of the relativistic formalism to inelastic scattering. As we discuss in a separate 
item, we are developing an effective interaction $t^{e f f}$ for use in standard distorted wave calculations as a simple means to incorporate the relativistic effects, i.e., it is not necessary to work with the complete set of invariant amplitudes which underlie the IA2 formalism. Good agreement of the $t^{e s f}$ based on the IA2 formalism with the phenomenological results of Kelly and collaborators encourages the belief that results for inelastic proton scattering by nuclei will be quite successful.

At this point it can be said that the relativistic impulse approximation does succeed to describe proton scattering data rather well. Off-shell effects are found not to produce important changes to the results based on a local potential in the Dirac equation. Recent work has examined the second-order correction to the relativistic optical potential and it has been found to be of minor importance. We have studied the Pauli-blocking corrections and found these also to be small, but significant at lower energies such as 200 $\mathrm{MeV}$. It remains to consider the effects of nuclear binding on the intermediate states of the $N N$ t-matrix and to consider the effects of full folding in the potential.

One of the most interesting and important questions is whether we can progress to a satisfactory linking of meson-exchange dynamics to the structure of hadrons. A key missing link of the hadronic approach to nuclear physics is a means to understand and calculate the vertex functions which are appropriate to hadronic interactions.

(S. J. Wallace, in Structure of Hadrons and Hadronic Matter, O. Scholten and J. H. Koch, eds., (World Scientific, Singapore) p. 84-113, 1991.)

\section{Relativistic and Off-Shell Effects in Elastic Proton Scattering.}

Recent progress on the relativistic and 'no-pair' analyses of proton scattering is reviewed in an invited talk in Taiwan which has been published in 1991. Excerpts from the article follow.

Relativistic descriptions of the nuclear force are possible within a meson exchange framework. In isoscalar channels, two basic features emerge, namely, strong scalar 
attraction at intermediate range and strong vector repulsion at short range. The scalar component of the force can be described by exchange of a scalar meson, $\sigma$, and the vector repulsion can be described by exchange of a vector meson, $\omega$. Feynman amplitudes associated with these meson-exchange interactions are

$$
\begin{gathered}
\mathcal{M}_{S}=\frac{g_{S}^{2}}{m_{S}^{2}+\vec{q}^{2}} \\
\mathcal{M}_{V}=-\gamma_{1} \cdot \gamma_{2} \frac{g_{V}^{2}}{m_{V}^{2}+\vec{q}^{2}}
\end{gathered}
$$

It is too simplified to describe all the scalar attraction as being due to exchange of a scalar meson. At least in part, the attraction arises from two-pion exchange interactions in which intermediate $N \Delta$ states are present. Coupled $N N, N \Delta$ and $\Delta \Delta$ channels are required to provide for such mechanisms and also to provide the dominant mechanism responsible for inelasticity in the $N N$ scattering namely, the excitation of $\Delta$ isobars and their subsequent decay. As we shall show, even when these refinements are taken into account the basic features of scalar attraction and vector repulsion remain.

The $N N$ interaction is described theoretically by a Feynman amplitude, $\mathcal{M}$, in quantum field theory. Since scattering involves two initial states and two final states and each nucleon state is a four-component Dirac spinor (with respect to a nucleon's center of mass motion), a complete description involves $4^{4}=256$ amplitudes. However parity invariance of the strong interaction eliminates half of these and so reduces the description to 128 parity invariant amplitudes. Time-reversal invariance further reduces the number of independent amplitudes to 72. Although not exact, charge symmetry (i.e., invariance with respect to interchange of neutrons and protons) is quite a good symmetry for the $N N$ interaction. If one neglects charge-symmetry violations, then a total of 56 amplitudes can be independent. In any case, a full description involves expansion in terms of scalar amplitudes times kinematical covariants.

In recent years, such complete descriptions of the $N N$ interaction have been developed based on meson-exchange models. Although the complete description involves many terms, the scalar attraction and vector repulsion mentioned above are still 
dominant features. Mean fields experienced by a nucleon in nuclear matter may be extracted using trace techniques as follows.

$$
\begin{gathered}
S=-\frac{1}{16} \operatorname{Tr}_{1} \operatorname{Tr}_{2}[\mathcal{M}] \rho \\
V=-\frac{1}{16} \operatorname{Tr}_{1} \operatorname{Tr}_{2}\left[\gamma_{1} \cdot \gamma_{2} \mathcal{M}\right] \rho
\end{gathered}
$$

where $\rho$ is the nuclear density. For scattering of $200 \mathrm{MeV}$ protons, one finds that the scalar interaction corresponds to about $300 \mathrm{MeV}$ of attraction in nuclear matter and the vector interaction corresponds' to aboit $200 \mathrm{MeV}$ of repulsion.

(S. J. Wallace, in Progress in Nuclear Physics, W-Y. P. Hwang, S-C. Lee, C-E Lee and D. J. Ernst, eds. (North Holland, Amsterdam, 1991), pp. 423-33.)

\section{Relativistic Analysis of the Deuteron and Elastic Electron-Deuteron Scattering}

We have obtained deuteron vertex functions by solving the quasipotential Dirac two-body equation,

$$
\Gamma\left(p_{\perp P} ; P\right)=\int \frac{d^{4} p^{\prime}}{(2 \pi)^{4}} V\left(p_{\perp P}, p_{\perp P}^{\prime} ; P\right) G_{0}\left(p^{\prime} ; P\right) \Gamma\left(p_{\perp P}^{\prime} ; P\right),
$$

with a one-boson exchange interaction using the parameters of the energy independent Bonn potential. The subscript $\perp P$ denotes the component of the variable perpendicular to the total momentum $P$, thus $p_{\perp P} \cdot \hat{P}=0$, with $\hat{P}=P / \sqrt{P^{2}}$. The quasipotential Dirac two-body propagator is:

$$
G_{0}(p ; P)=i g_{0}(p ; P) 2 \pi \delta(p \cdot \hat{P})
$$

with

$$
\begin{gathered}
g_{0}(p ; P)=\sum_{\rho_{1}, \rho_{2}} \frac{\Lambda_{1}^{\rho_{1}}\left(p_{1 \perp P}\right) \Lambda_{2}^{\rho_{2}}\left(p_{2 \perp P}\right)}{\rho_{1}+\rho_{2} E_{2}-\epsilon_{1}-\epsilon_{2}}, \\
\Lambda_{i}^{\rho_{i}}\left(p_{i \perp P}\right)=\frac{1}{2 \epsilon_{i}}\left[\rho_{i} \epsilon_{i} \gamma_{i} \cdot \hat{P}+\gamma \cdot p_{i \perp P}+m_{N}\right],
\end{gathered}
$$


and

$$
\epsilon_{i}=\sqrt{m_{N}^{2}-p_{i \perp P}^{2}}
$$

Note that $p_{i \perp P}^{2}<0$. For equal masses $E_{1}=E_{2}=M_{D} / 2$. The quasipotential equation is covariant and the vertex functions can be boosted to any frame, but will only be known on the constraint $p \cdot \hat{P}=0$.

Using these vertex functions the electomagnetic form factors for elastic electron-deuteron scattering have been calculated. The impulse approximation to the current in the Breit frame is:

$$
\begin{aligned}
\left\langle J_{I A}^{\mu}\right\rangle= & \int \frac{d^{3} p_{B}}{(2 \pi)^{3}} \bar{\Gamma}\left(p_{\perp P c m}^{\prime} ; P^{c m}\right) g_{0}\left(p_{\perp p c m}^{\prime} ; P^{c m}\right) \Lambda_{L 1}^{\prime} \Lambda_{L 2}^{\prime} \\
& \hat{\rho}_{2} \gamma_{2}^{0}\left(J_{1}^{\mu}+J_{2}^{2}\right) \Lambda_{L 1} \Lambda_{L 2} g_{0}\left(p_{\perp P c m} ; P^{c m}\right) \Gamma\left(p_{\perp P^{c m}} ; P^{c m}\right),
\end{aligned}
$$

where $P^{\mathrm{cm}}=\left(M_{D}, 0\right)$. The one body electromagnetic form factor for particle one and a photon of momentum $q=\left(0,0,0, q_{3}\right)=p_{1}^{\prime}-p_{1}$ is

$$
\begin{gathered}
J_{1}^{\mu}=F_{1}(q) \gamma_{1}^{\mu}+F_{2}(q) \frac{q_{3}}{2 m_{N}} \sigma_{1}^{\mu \nu} q_{\nu}, \\
\hat{\rho}_{2}=\frac{\alpha_{2} \cdot \mathbf{p}_{2}+\beta m_{N}}{\epsilon\left(\mathbf{p}_{2}\right)},
\end{gathered}
$$

and $\Lambda_{L 1}\left(\Lambda_{L 1}^{\prime}\right)$ and $\Lambda_{L 2}\left(\Lambda_{L 2}^{\prime}\right)$ are the one particle boost operators between the Breit frame and the initial (final) deuteron center of momentum frame.

Unfortunately the quasipotential constraints for the initial and final states, namely $p_{\perp P} \cdot \hat{P}^{\prime}=0$ and $p_{\perp P^{\prime}}^{\prime} \cdot \hat{P}^{\prime}=0$, cannot be satisfied along with four-momentum conservation at the photon which requires $q=P^{\prime}-P$ and $q / 2=p^{\prime}-p$. A consequence is that the relative four-momentum variables required in the initial and final states do not satisfy the quasipotential constraints. Calculations have shown that different approximations of the Lorentz transformation equations yield significant differences for the form factors. Using the quasipotential vertex functions which satisfy the quasipotential constraints but neglecting the time-components of relative four-momenta implied by momentum conservation yields a good description of the data. 
Work on treating the constraints consistently with four-momentum conservation is in progress. The constraint of the quasipotential vertex function can be altered using

$$
\Gamma\left(p_{\perp P_{b}} ; P\right)=\int \frac{d^{4} p^{\prime}}{(2 \pi)^{4}} V\left(p_{\perp P_{b}}, p_{\perp P}^{\prime} ; P\right) G_{0}\left(p_{\perp P}^{\prime} ; P\right) \Gamma\left(p_{\perp P}^{\prime} ; P\right)
$$

Where, for example, $P_{b}=\left(P^{\prime}+P\right) / 2$. This equation is formally correct, but if the kernel is approximated by the one boson exchange potential, the potential contains unphysical singularities due to the quasipotential constraints. Such singularities are not present if the full kernel is kept. We expand the potential in a Taylor series about zero retardation to approximate the case where the full kernel is used.

The meson exchange currents ( $\rho \pi \gamma, \omega \sigma \gamma$, and $\omega \eta \gamma)$ are approximated by

$$
\begin{aligned}
\left\langle J_{M E C}^{\mu}\right\rangle= & \int \frac{d^{3} p^{\prime} d^{3} p}{(2 \pi)^{6}} \bar{\Gamma}\left(p_{\perp P c m}^{\prime} ; P^{c m}\right) g_{0}\left(p_{\perp P}^{\prime} ; P^{c m}\right) \Lambda_{L 1}^{\prime} \Lambda_{L 2}^{\prime} \\
& \Gamma_{M E C}^{\mu} \Lambda_{L 1} \Lambda_{L 2} g_{0}\left(p_{\perp p c m} ; P^{c m}\right) \Gamma\left(p_{\perp P c m} ; P^{c m}\right),
\end{aligned}
$$

with $\Gamma_{M E C}^{\mu}$ given by the appropriate terms for the meson nucleon vertices, the different meson propagators, and the meson-photon-meson vertex. The meson propagators are approximated by setting their retardation to zero. One of the $\phi$ angles is integrated analytically and the other five variables are integrated numerically using quadurature.

Results of calculations are shown in the figure.

Finally we plan to calculate the deuteron vertex function and electromagnetic form factors using a potential with a softer $\pi N N$ vertex form factor, which is motivated by quark models. This work is the Ph.D. thesis research of Neal Devine.

(N. Devine and S. J. Wallace)

\section{E. Effective Interaction Based on the Relativistic IA2 Formalism}

Although progress has been made in developing a complete set of Lorentz-invariant $N N$ amplitudes and the IA2 optical potential constructed from them has provided successful descriptions of elastic scattering data, there have been no 

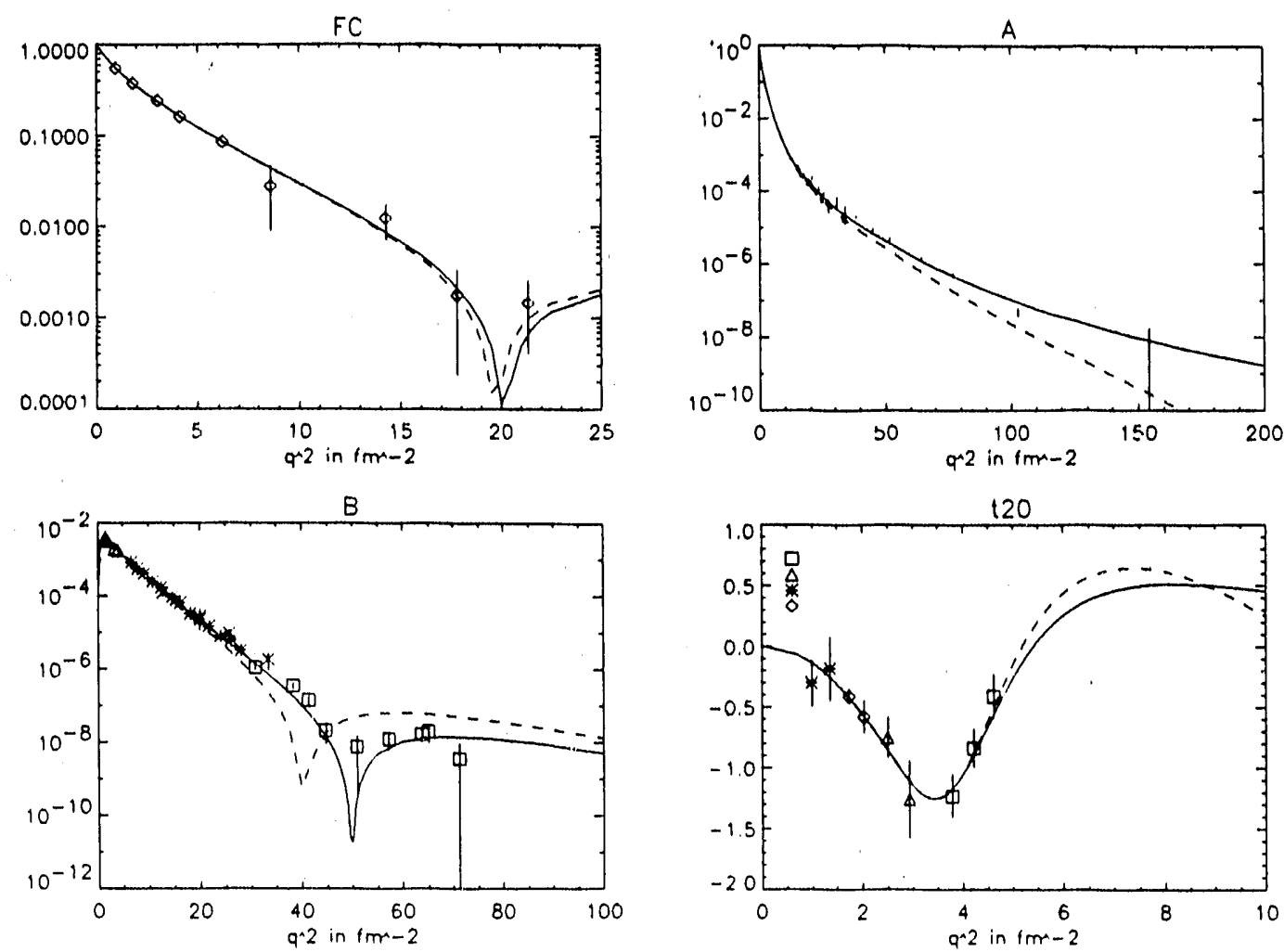

Fig. 1 Deuteron form factors: charge, A, B, and t20. The dashed line is the IA contribution. The solid line includes the meson exchange current contributions with $g_{\rho \pi \gamma}=.563, g_{\omega \sigma \gamma}=-.32$, and $g_{\omega \eta \gamma}=-.206$.

applications to inelastic scattering. The reason is that a great deal of work is required to develop new computer codes to do inelastic calculations. Some Dirac distorted wave calculations and Dirac coupled channel calculations have been reported. However, all analyses to date employ a schematic interaction based on a minimal set of five $N N$ amplitudes. A complete description requires 44 amplitudes as in the IA2 analysis.

A different approach to this problem is to incorporate the effects of the complete set of $N N$ amplitudes into an effective interaction which may be used in standard DWIA codes. Substantial progress has been made on this alternative approach in the past year and a first paper is in preparation.

Recent work by Kelly and collaborators has developed a phenomenological effective interaction for proton-nucleus scattering. Simultaneous fits of inelastic and elastic scattering data were made and it was found that data for a broad range of nuclei 
could be fit with a single density-dependent effective interaction. An important ingredient is the so-called rearrangement effect which causes increased density dependence of the inelastic effective interaction over that of the elastic effective interaction. Moreover the Schrödinger equivalent optical potentials deduced from the phenomenology are in close agreement with those of the relativistic IA2 formalism developed at Maryland.

The effective interaction based on the relativistic IA2 formalism is deduced as follows. Solution of the Dirac equation with a potential constructed from a complete set of Lorentz invariant nucleon-nucleon amplitudes is shown to be equivalent to solution of the relativistic Schödinger equation with a potential based on a density-dependent effective interaction. The effective interaction for elastic scattering is defined as a matrix element of the Lorentz invariant nucleon-nucleon interaction $\hat{\mathcal{M}}$ where the matrix element differs from the free t-matrix in that distortions are present in three of the Dirac spinors.

Distorted Dirac wave functions, $\psi$, are described as follows.

$$
\begin{aligned}
\psi & =\left[u^{+}+u^{-} \frac{1}{E+E(p)-U^{--}} U^{-+}\right] \psi^{+} \\
& =\Omega_{1} u^{+} \psi^{+}
\end{aligned}
$$

where $\psi^{+}$is a Schrödinger-Pauli wave function and $\Omega_{1}$ is a Moller operator defined as follows,

$$
\Omega_{1}=1+u^{-} \frac{1}{E+E(p)-U^{--}} \bar{u}^{-} \hat{U}
$$

The Moller operat or links the full Dirac wave function to the Schrödinger-Pauli function $\psi^{+}$. The distorted wave involves an admixture of positive- and negative-energy spinors such as would occur due to couplings to virtual $N \bar{N}$ states in analogy with QED. However the distorted spinors for a composite nucleon may have a different dynamical origin, as has been demonstrated by considering the propagation of a nucleon in nuclear matter using QCD sum rule techniques. The argument is quite simple in essence. Lorentz invariance requires a nucleon in free space to have two parts to its wave function which are linked as are the large and small componenis of a Dirac spinor. In uniform nuclear matter, the form of the spinor must be similar, differing only because the energy and 
mass parameters are allowed to differ in the nuclear medium. However, the energy is known to be close to its free value as the nuclear binding is only about $16 \mathrm{MeV}$ per nucleon. The mass is determined by the dynamics and it is estimated to be several hundred $\mathrm{MeV}$ less than the free mass. The implication is that there are cancelling scalar and vector interactions which is the same result that is found when the $N N$ interaction is described by Lorentz-invariant amplitudes. In the QCD sum rule analysis, the dynamical origin of the decreased mass is the decrease of the quark condensates of QCD. In general, the distorted waves defined above may be thought of as due to the self energy which alters the nucleon's mass in a nuclear medium.

Using the distorted spinors from the relativistc IA2 formalism, the effective interaction for elastic scattering is given by the expression

$$
t_{e f f}^{e l}\left(\vec{p}, \vec{p}_{2} \rightarrow \overrightarrow{p^{\prime}} \vec{p}_{2}^{\prime}\right) \equiv-\bar{u}^{+}\left(\vec{p}^{\prime}\right) \bar{u}_{2}^{+}\left({\overrightarrow{p^{\prime}}}_{2}\right) \bar{\Omega}_{2} \hat{\mathcal{M}}\left(p, p_{2} \rightarrow p^{\prime}, p_{2}^{\prime}\right) \Omega_{1} \Omega_{2} u^{+}(\vec{p}) u_{2}^{+}\left(\vec{p}_{2}\right)
$$

A similar reduction of the relativistic distorted wave impulse approximation for inelastic scattering is performed to obtain an inelastic effective interaction which differs from the elastic one in that distortions are present in all four Dirac spinors. The extra distortion is related to the so-called rearrangement effect. The interaction takes the form

$$
t_{e f f}^{\text {inel }}\left(\vec{p}, \vec{p}_{2} \rightarrow \vec{p}^{\prime} \vec{p}_{2}^{\prime}\right) \equiv-\vec{u}^{+}\left(\overrightarrow{p^{\prime}}\right) \bar{u}_{2}^{+}\left(\vec{p}_{2}^{\prime}\right) \bar{\Omega}_{1} \bar{\Omega}_{2} \hat{\mathcal{M}}\left(p, p_{2} \rightarrow p^{\prime}, p_{2}^{\prime}\right) \Omega_{1} \Omega_{2} u^{+}(\vec{p}) u_{2}^{+}\left(\vec{p}_{2}\right)
$$

Due to the distorted waves, there is substantial density dependence in the effective interactions, somewhat more so for the inelastic effective interaction. A local density approximation is used to remove nonlocalities and thereby to approximate the effective interactions. Results at $200 \mathrm{MeV}$ for the central, spin independent component of the interaction are shown in the figure.

For elastic scattering, a reasonable reproduction of the equivalent IA 2 results is obtained using the elastic effective interaction in the relativistic Schrödinger equation. The IA 2 interaction is similar to a phenomenological effective interaction obtained by Kelly et al. by fitting experimental data for elastic and inelastic scattering. In this respect, the relativistic effective interaction seems to be superior to other effective 


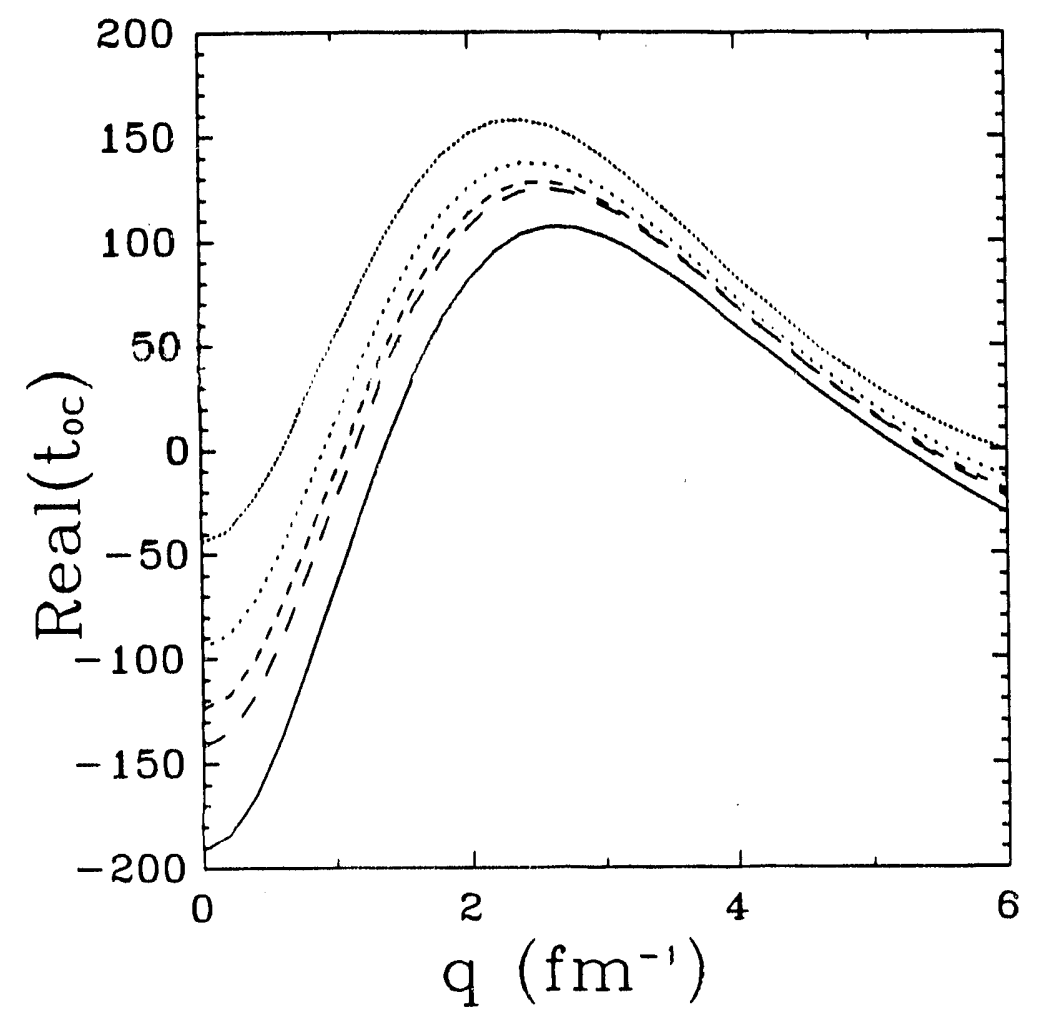

Fig. 1 Real part of the central, spin-independent central t-rratrix at five values of the nuclear density are plotted versus momentum transfer, $q$. The free $t$-matrix is shown by the solid line. The other lines show results for $\frac{1}{4}, \frac{1}{2}, \frac{3}{4}$ and full nuclear matter density.

interactions in the literature, particularly for energies near $500 \mathrm{MeV}$. The inelastic effective interaction may be used in conventional analyses based on the distorted-wave impulse approximation. It provides a simple method for incorporating a complete set of $N N$ amplitudes in conventional analyses. The full spin and isospin dependence of the inelastic effective interaction is being developed and a paper is in preparation.

(R. J. Furnstahl and S. J. Wallace)

\section{F. Full Folding Optical Potential Analysis}

Some years ago, nonrelativistic approaches to elastic proton scattering did not produce successful descriptions of experimental data based on the impulse approximation. Since relativistic analyses were significantly more successful, particularly with respect to 
describing spin observables, the nonrelativistic approach was considered to be failing. However several approximations are used in the theoretical analyses which kept the subject open. A very interesting result was found when the first full-folding calculations were done based on the nonrelativistic formalism. Predictions for elastic scattering near $200 \mathrm{MeV}$ were dramatically improved both with regard to the description of the cross section and the spin observables.

Some conflicting claims have also appeared in the literature. The conflict centers on whether the energy at which one evaluates the t-matrix in the $\rho$ form of the optical potential varies as one integrates over the motion of the struck nucleon. When the energy variable is fixed at an optimal point, one obtains a factorized form of the optical potential which is much more convenient for analysis. Analyses of the off-shell effects using a factorized optical potential have been performed by three groups. One group found them to be quite large and also such as to improve the agreement with data at energies near $200 \mathrm{MeV}$ (but not at $500 \mathrm{MeV}$ ). Two other groups found minor effects due to off-shell variations of the t-matrix within the factorized potential. Our results on this subject show that the off-shell effects should be minor when the boost of the t-matrix from the laboratry frame to the $N N \mathrm{~cm}$ frame is done in a manner consistent with obtaining the correct on-shell energy in the $N N \mathrm{~cm}$ frame. We have found that the on-shell t-matrix dominates the scattering and this can be understood in simple terms from the suppression of contributions to the $K$-matrix from momenta which are more than $1 F^{-1}$ off-shell due to the rapid decrease of the nuclear form factor.

Accepting the result that momentum dependence of the t-matrix is of minor importance means that the major part of the full-folding improvement must come either solely from the variations of the energy of the t-matrix or some complicated interplay of the energy variation with off-shell mornentum dependence, barring any errors in the admittedly complex calculations. It is not clear from the full-folding calculations which is the case as everything is done at once. Moreover no independent check of the full-folding results has been reported. 
solution. One issue which arises may be studied in a much simpler context, namely, three body bound states using a local potential omitting spin. In the simplest form of the problem, one has in the rest frame

$$
\begin{array}{r}
\psi\left(\vec{p}_{1}, \vec{p}_{2},-\vec{p}_{1}-\vec{p}_{2}\right)=G_{0}\left(\vec{p}_{1}, \vec{p}_{2},-\vec{p}_{1}-\vec{p}_{2}\right)+\int \frac{d^{3} q}{(2 \pi)^{3}} V(q)\left[\psi\left(\vec{p}_{1}-\vec{q}, \vec{p}_{2}+\vec{q},-\vec{p}_{1}-\vec{p}_{2}\right)\right. \\
\left.+\psi\left(\vec{p}_{1}-\vec{q}, \vec{p}_{2},-\vec{p}_{1}-\vec{p}_{2}+\vec{q}\right)+\psi\left(\vec{p}_{1}, \vec{p}_{2}+\vec{q},-\vec{p}_{1}-\vec{p}_{2}-\vec{q}\right)\right] .
\end{array}
$$

Direct numerical solutions of a form of this equation have been reported by Kalusch and Glockle (Z. Phys.) using the nonrelativistic propagator

$$
G_{0}\left(\vec{p}_{1}, \vec{p}_{2}-\vec{p}_{1}-\vec{p}_{2}\right)=\frac{1}{E-\left(p_{1}^{2}+p_{2}^{2}+\left(\vec{p}_{1}+\vec{p}_{2}\right)^{2}\right) /(2 M)} .
$$

where $E$ is the binding energy $(E<0)$. The analysis of Kalusch and Glockle uses Jacobi coordinates such that one of the three integrations may be done analytically. To avoid any restriction to nonrelativistic kinematics, the full three-dimensional integration is done numerically in our work. Direct integration of the three-particle equation is known to involve singularities arising from the noncompact kernel. For some cases of interest, it is nevertheless possible to obtain a solution for the ground state of the system.

We have developed a computer code which reproduces the results of Kalusch and Glockle and we are extending the analysis by replacing the nonrelativistic propagator by one involving relativistic kinetic energies as follows

$$
G_{0}\left(\vec{p}_{1}, \vec{p}_{2},-\vec{p}_{1}-\vec{p}_{2}\right)=\frac{1}{3 M+E-\epsilon\left(p_{1}\right)-\epsilon\left(p_{2}\right)-\epsilon\left(\left|\vec{p}_{1}-\vec{p}_{2}\right|\right)}
$$

where $\epsilon(p)=\sqrt{p^{2}+M^{2}}$. This propagator is appropriate to the rest frame of the bound state in the relativistic quasipotential formalism when only positive-energy states are kept.

In the relativistic case, the propagator cannot be diagonalized by use of the Jacobi momenta and this is a generic feature of relativistic dynamics. In a partial wave analysis, it presents an important complication. However in the direct numerical solution, there is no penalty for use of the relativistic form of the kinetic energy. Our plan is to solve the problem directly using gaussian integration, a spline interpolation algorithm and an 
iterative solution for the bound state. Initial work will be done on a DECStation 5000 with $40 \mathrm{Mb}$ of memory to discover the feasibility of a much larger calculation in which spin-dependence and a realistic interaction are taken into account. As a byproduct, the initial investigation will provide interesting results for the relativistic corrections.

(S. J. Wallace and A. Delfina)

\section{Heavy Ion Dynamics and Related Processes}

\section{A. Overview}

During the 1991-92 academic year, Prof. Griffin's research has been devoted even more heavily to the study of the " $\left(e^{+} e^{-}\right)$Puzzle" posed by the sharp lepton pairs observed at the GSI laboratories. The immediate goal is to continue the development of an explicit phenomenological description of the data which is based upon the assumption that the leptons arise from the decay of some hitherto unknown composite particle which exhibits perhaps a substantial component of "Quadronium", the $\left(e^{+} e^{+} e^{-} e^{-}\right)$leptonic atom. Last year, the successful description of the EPOS sum and difference energy distributions and its implication that the composite particle niay decay either bound to a nuclear ion or isolated in free space, provided a basis for inferring the polarization properties of the C-particle. These now allow us to proceed to the study of the dynamics of the two heavy ions plus the $C\left(Q_{0}\right.$ ?) -particle system which follow the spontaneous creation of the "C". The results promise to provide some of the details required to analyze the data more closely, and especially the relationship between the data of the Orange and EPOS groups.

As noted above, our phenomenology indicates that the neutral composite C-particle sometimes binds to an emergent high- $Z$ nuclear ion by polarization forces, and subsequently decays into $\left(e^{+} e^{-}\right)$under the influence of the nuclear Coulomb field. The inference was immediately drawn that if such bound decays occur, then they must imply a new process of Sharp Annihilative Positron Emission (SAPosE) which yields a series of sharp positron lines with energies in the range $950>\mathrm{E}_{+}>600 \mathrm{keV}$. Our calculated line 
widths and intensities for this new SAPosE process were published last year.

Furthermore, three additional decay modes (the One Photon, Tri-Lepton and Bi-Positron Emissions) have now been recognized all of which are forbidden for the isolated $C\left(Q_{0}\right.$ ?) particle, but allowed in principle for $C\left(Q_{0}\right.$ ?) bound to a nucleus.

This year has also provided progress in a new unexpected direction not anticipated in last year's proposal. In particular, we are now able to reject certain widely accepted limits upon the lifetime of the decaying composite particle allegedly imposed by the Bhabha scattering data. A careful reconsideration of the basis for these claims shows that they are artifacts of the theoretical model-dependent assumptions of the analysis and not compelling conclusions of the Bhabha data itself. As outlined in our companion proposal for research during 1992-93, several aspects of the $C\left(Q_{0}\right.$ ?) -particle-phenomenology seem at present to offer excellent promise for further progress in the near term.

During the last year, work continued also in the theory of heavy ion reactions, directed especially towards the confrontation of the deep inelastic $\mathrm{N}$ and $\mathrm{Z}$ drifts with calculations based upon the "permeation" current. We expect soon to complete our survey comparison of our theory and the extensive body of relevant deep inelastic data.

\section{B. Leptonic Emissions from High-Z Heavy Ion Collisions \\ 1. The Composite Particle $\left(Q_{0}\right.$ ?) Senario for the GSI Lines, and Bound Decays}

The Composite Particle $\left(Q_{0}\right.$ ? $)$ Scenario for the GSI $\left(e^{+} e^{-}\right)$lines is able to provide a complete qualitative framework for describing the sharp leptonic pairs produced in high-Z heavy ion collisions (Int. J. Mod. Phys. A6, 1985, (1991)). It suggests also when compared with the EPOS data that the composite particle sometimes remains bound from its creation to one of the nuclear ions, exhibiting therefore the effects of the nuclear Coulomb field in its decay energy distributions (Phys. Rev. Lett. 66, 1426 (1991)). Such binding implies in turn the possibility of the new process of Sharp Annihilative Positron Emission discussed in quantitative detail in last year's report. However, three additional 
decay modes, each of which is forbidden for the composite particle in free space, become possible when $C\left(Q_{0}\right.$ ?) is bound to a nucleus, as we now discuss (Proc. 6th Int. Conf. on Nuclear Reaction Mechanisms, Varenna, June 1991, ed. by E. Gadioli, U. of Milan, p. $758)$.

\section{(J. J. Griffin)}

\section{One Photon Decay of Composite $C\left(Q_{0}\right.$ ?) Particle}

An isolated composite particle which decays into an $\left(e^{+} e^{-}\right)$pair is forbidden by energy-momentum conservation from decaying into a single photon. But if it is bound to a heavy object, then it may honor momentum conservation with only a negligible shift and a negligible spread in its energy. It follows that decay photons with energies nearly equal to each sharp eigenenergy of the decaying system can be expected to occur in the decay spectrum. Excess photons are indeed seen in the energy region near $1.8 \mathrm{MeV}$, but so far with resolution too poor to determine whether any of them have nearly sharp energies equal to the same sharp decay energies observed for the $\left(e^{+} e^{-}\right)$pairs. Unfortunately nuclear gamma rays are also expected in the energy region, so that the mere presence of a poorly resolved excess does not show that the one-gamma decay has actually been observed.

\section{(J. J. Griffin)}

\section{Bi-Positron (BiPE) Emittive Decay of $C\left(Q_{0}\right.$ ?)}

A second new decay mode which can also occur when the $C\left(Q_{0}\right.$ ? $)$ is bound to a nucleus is the Bi-Positron Emittive Decay (BiPE). This is a decay in which two electrons are deposited into unfilled Bohr orbits and two positrons are ejected with the remaining available energy. It is therefore closely analogous to the Sharp Annihilative Positron (SAPosE) Emission, except that it presumes the decay to occur from a "Quadronium" $\left(e^{+} e^{+} e^{-} e^{-}\right)$component of the composite $C\left(Q_{0}\right.$ ?) particle state. Its observation could therefore provide evidence of the probability that the composite particle is, at any given 
time, in fact a quadronium-like object. It is therefore remarkable for the fact that it speaks to the internal structure of the particle (as none of the presently available data do under the $C\left(Q_{0}\right.$ ?) Scenario).

We note also that Bi-Positronium Emission can occur only if the composite eigenenergy exceeds $1.780 \mathrm{MeV}$ : only then can the two emitted positrons have a positive kinetic energy, even when both of the electrons are captured into the most tightly bound $1 \mathrm{~s}$ orbits of $\mathrm{U}$. This threshold allows the process to occur for the $805 \mathrm{keV}$ lines, but forbids it for the $620 \mathrm{keV}$ line of EPOS. In each case, the positrons have very low energies.

(J. J. Griffin)

\section{Tri-Lepton (TriLE) Emittive Decay of $C\left(Q_{0}\right.$ ?)}

Analogous to the Bi-Positron Decay is the Tri-Lepton Emission, in which a Quadronium component of the $C\left(Q_{0}\right.$ ? $)$ eigenstate decays by depositing one electron into a Bohr orbit and emitting one electron and two positrons. This process requires a decaying eigenenergy in excess of $1.912 \mathrm{MeV}$. It is therefore forbidden for all three well-establihsed EPOS lines $(805,748$ and $620 \mathrm{keV})$. Its observation would therefore signal a higher-lying eigenstate with a substantial "Quadronium" component in its wave function.

(J. J. Griffin)

\section{Occurence of Bound Decays for U+Ta, Not for U+Th}

We note also that the $C\left(Q_{0}\right.$ ?) Scenario provides an immediate qualitative understanding of why the $\mathrm{U}+\mathrm{Ta}$ collision should yield $C\left(Q_{0}\right.$ ?) bound to an ion while $\mathrm{U}+\mathrm{Th}$ yields free $C\left(Q_{0}\right.$ ?) decays. Such behavior follows qualitatively from the fact that the potential felt by the neutral $C\left(Q_{0}\right.$ ?) particle is due to its polarization by the electric field of the nuclei. As the two ions separate this field becomes small over a growing volume between them, and the resulting forces along the line of nuclear centers become proportional to the square of the difference in the ionic charges, $\left(Z_{1}-Z_{2}\right)^{2}$. Since this is $\sim(19 / 2)^{2}$ larger for U-Ta than for U-Th, it is no wonder that the U-Ta combination more 
efficiently accelerates the $C\left(Q_{0}\right.$ ?) into a bound state with the $\mathrm{U}$ ion.

This picture also supplies the unambiguous implication that $C\left(Q_{0}\right.$ ?) is in fact bound to $\mathrm{U}$ and not to $\mathrm{Ta}$, as well as other specific implications about the velocity distributions of the $C\left(Q_{0}\right.$ ?) which decay free. We hope soon to quantify some of these implications.

(J. J. Griffin)

\section{Alleged Constraints on the Composite $\left(Q_{0}\right.$ ?) Particle Lifetime from Bhabha Scattering Data are False}

Recently' Tsertos et al. published (in Phys. Lett. B266, 259 (1991)) the following claim: "Our conclusive results rule out theoretical proposals that involve neutral particles or $\left(e^{+} e^{-}\right)$bound states in this $\left(\sim 1.8 \mathrm{MeV} / \mathrm{c}^{2}\right)$ mass range as an explanation of the $\left(e^{+} e^{-}\right)$lines observed at GSI". If valid, this claim would foreclose the composite particle scenario which we believe most adequately comprehends the data of this " $\left(e^{+} e^{-}\right)$-Puzzle".

We therefore have carried out a review of the Bhabha measurements and analyses which lead to this claim. We find that in fact the lifetime inferences upon which this conclusion is based are not implied by the Bhabha data at all, but are instead artifacts of the authors' assumption that only the one $\left(e^{+} e^{-}\right)$decay channel is possible. This paper will soon be submitted for publication.

(J. J. Griffin)

\section{Alleged Limits on $C\left(Q_{0}\right.$ ?) Lifetime from ( $\left.e^{-2}\right)$ Apply Only to Elementary $\mathbf{X}_{0}$ Particles, Not to Composites}

In our study of the assertions based upon Bhabha data, we were led also to consider the limits upon the particle lifetime which high precision theoretical quantum electrodynamics is alleged to impose (Reinhardt, et al., Phys. Rev. C33, 194 (1986)). We find that the calculations upon which these claims are based assume a simple coupling term between the hypothetical $\mathrm{X}_{0}$ particle field and the lepton $\left(e^{+} e^{-}\right)$fields to which it 
decays. If the $\mathrm{X}_{0}$ particle were elementary, this description then might be considered correct in principle, and the (unknown) coupling constant, $\alpha_{x}$, could be taken as a fundamental characteristic of the $\mathrm{X}_{0}$-field. Then the change which it affects in the calculated value of $\left(\mathrm{g}_{e}-2\right)$ could place a valid upper bound upon $\alpha_{x}$.

But if $X_{0}$ is not elementary, but composite, then the same coupling term must be considered as an effective coupling, which yields valid results only when applied at the tree level. The suggestion that these calculations can be applied as well for a composite as for an elementary particle is therefore in error.

We conclude therefore that, despite published assertions to the contrary, no valid limits, either theoretical or experimental (except for the upper bound, $\tau<-10^{-10}$ sec., imposed by the size of the fiduciary volume in the Orange experiment) exist at present.

(J. J. Griffin)

\section{Fundamental Origins of Quadronium Binding Energies}

At the fundamental level, we continue to prefer the conservative assumption that Quadronium can perhaps be bound, even so strongly as it is, by the well-known forces of quantum electrodynamics (QED) alone, despite the fact that we can as yet offer no specific mechanism for such binding. Therefore, our efforts are directed towards a search within QED for a binding mechanism which is specifically characteristic of the four-lepton system. We also recognize full well that neither the relativistically bound two-body problem, nor the nonrelativistic four-body problem are well comprehended by present theory. It would seem to follow that the relativistically bound four-body problem is formidable, and will not soon be fully solved.

(J. J. Griffin) 


\section{Theoretical Studies of Heavy Ion Dynamics}

\section{Permeation Current and $\bar{N}, \bar{Z}$ Drifts in Heavy Ion Collisions}

A survey of the theoretical and experimental aspects of the $\bar{N}, \bar{Z}$ drifts in deep inelastic collisions is nearing completion. It shows that the new quantal permeation current allows a significant improvement both qualitatively and quantitatively in the agreement between the calculated and observed $\bar{N}, \bar{Z}$ drifts.

(T. Kiess, M. Dworzecka and J. J. Griffin ) 


\section{Nuclear Theory Group Personnel}

Faculty

M. K. Banerjee

T. D. Cohen

J. J. Griffin

W. M. MacDonald*

E. F. Redish*†

S. J. Wallace
Visiting Faculty

W. Broniowski* (til 9/92)

A. Delfino*

M. Dworzecka*

G. Rupp* (til 2/92)
Research Associates

H. Forkel

Y. Koike (til 9/92)

D. Leinweber

J. Milana (as of 9/92)

H. J. Lu (as of $9 / 92$ )
Research Graduate Students

C. Edward Bell (Ph.D. exp. 1992)

Neal Devine (Ph.D. exp. 1992)

Xuemin Jin

Myunggyu Kim

Edward Smith-Rowland (Ph.D. exp. 1992)

Randloph Wild* $\underline{\text { Summer Research Graduate Students }}$

Mountaga Aw

Ernest Barreto

Paul Dulany

Paul Lynch

Summer Undergraduate Student

Carl Schroeder

* No salary support from DOE grant.

†On sabbatical leave Aug. 1992-Sept. 1993.

The Group's activities are supported by one full-time secretary (Loretta

Robinette). Mrs. Sylvia Doggett retired at the end of February, 1992.

In connection with our seminar program, our research collaborations, our interviewing of postdoctoral candidates and in order to bring leading theorists to Maryland, we have an active visitor program. All of our visitors in the past year are listed below: 
NAME, AFFILIATED INS'TITUTION, AND DATES OF VISIT

$\underline{1991}$

Mr. Hong Jung, Carnegie Mellon University: 2 days (1/22-23/91)

Mr. Niels Walet, University of Pennsylvania: 1 day (1/30/91)

Dr. Fred Myhrer, University of South Carolina: 2 days (2/5-6/91)

Mr. Hui-Wen Wang, Brooklyn College: 2 days (2/7-8/91)

Dr. B. Mueller, Duke University: 2 days (2/13-14/91)

Mr. Dao Chen Zheng, Rutgers University: 2 days (2/14-15/91)

Mr. Eric Swanson, University of Toronto, Canada: 3 days (3/5-7/91)

Dr. Carl Carlson, College of William \& Mary: 1 day (3/13/91)

Dr. Derek Leinweber, TRIUMF, Vancouver: 5 days (3/14-18/91)

Dr. E. Shuryak, SUNY at Stony Brook: 1 day (3/20/91)

Mr. Charles Albert, Texas $A \& M$ University: 2 days (4/1-2/91)

Dr. Anatoly Radyushkin, CEBAF: 2 days (4/3-4/91)

Dr. Richard Silbar, Los Alamos National Lab. \& D.O.E.: 1 day (4/5/91)

Dr. Peter Lepage, Cornell University: 1 day (4/19/91)

Dr. Victor Flambaum, Novosibirs: 1 day $(4 / 29 / 91)$

Dr. Ishmuel Zahed, SUNY at Stony Brook: 1 day (5/1/91)

Dr. Nathan Isgur, CEBAF, Newport News: 1 day (5/8/91)

Dr. Martin Lavelle, University of Regensburg, Germany: 1 day (6/13/91)

Dr. Shmuel Nussinov, Tel-Aviv University, Israel: 5 days (7/8-12/91)

Dr. Su Houng Lee, Yonsei University, Korea: 13 days (7/8-20/91)

Dr. Nafteli Auerbach, Tel-Aviv University, Israel: 7 days (9/3-9/91)

Dr. Nikolaya N. Nikolaev, Landau Institute, Moscow, USSR: 2 days (9/9-10/91)

Dr. Joseph A. Carlson, Los Alamos National Lab: 1 day (9/18/91)

Dr. Bojan Golli, Ljebbljana, Yugoslavia: 2 days (9/30-10/1/91)

Dr. Caio Lewenkopf, Max-Planck Institute, Heidelberg: 4 days (10/7-10/91)

Dr. Jonathan Engel, Bartol Institute: 1 day (10/23/91) 
Dr. Hiroshi Ito, George Washington University: 1 day (10/30/91)

Dr. Hans Pirner, University of Heidelberg, Germany: 2 days (11/12-13/91)

Dr. Mikhail Eides, Leningrad Inst. of Nuclear Physics: 2 days (11/26-27/91)

Dr. Winston Roberts, CEBAF, Newport News, Virginia: 2 days (11/16-27/91)

Dr. Marl: Strikman, Pennsylvania State University: 2 days (11/26-27/91)

Dr. Robert Pisarski, Brookhaven National Lab: 2 days (12/3-4/91)

Dr. Gerald E. Brown, SUNY at Stony Brook: 1 day (12/11/91)

Dr. Leonid Frankfurt, Michigan State University: 3 days (1/28-30/92)

Mr. Wai-Keung Tang, Columbia University: 2 days (2/4-5/92)

Mr. Veseteinn Thorsson, SUNY at Stony Brook: 2 days (2/11-12/92)

Dr. Joseph P. Milana, College of William \& Mary: 2 days (2/18-19/92)

Mr. Anand Subbaraman, Syracuse University: 2 days (2/25-26/92)

Mr. Hung Jung Lu, Stanford University: 3 dan's (3/1-3/92)

Mr. Grigorios I. Poulis, MIT, Cambridge: 1 day (3/4/92)

Mr. Christian Weiss, SUNY at Stony Brook: 1 day (3/13/92)

Dr. Zbigniew Dziembowski, Temple University: 1 day (3/25/92)

Dr. Boris Ioffe, ITEP, Moscow, USSR: 28 days (3/31-4/29/92)

(Visited both the Nuclear Theory and Elementary Particle Groups)

Dr. Xiang Dong Ji, MIT, Cambridge: 1 day (4/1/92)

Dr. Hong Jung, Carnegie Mellon University: 1 day (4/3/92)

Dr. Martijn Dekker, University of Utrecht, Netherlands: 2 days (4/16-17/92)

Dr. Mannque Rho, SPT/CEA. Saclay, France: 2 days (4/27-28/92)

Dr. Eugene Drukarev, Leningrad Inst. of Nuclear Physics: 8 days (5/11-19/92)

\section{LONGER-TERM VISITORS}

Ur. John A. Tjon, University of Utrecht, The Netherlands, 1991 \& 1992

Dr. Georges Rupp, CFN/INIC, Lisbon, Portugal, 1991-92

Dr. Antonio Delfino, Universidade Federal Fluminense, Brazil, 1991-93 
Dr. Wojciech Broniowski, Inst. of Nuclear Physics, Krakow, Poland, 1991-92

Dr. Brian Serot, Indiana University, 1992

\section{PERIODIC VISITORS}

Dr. Maria Dworzecka, George Mason U-: ersity, Fairfax, Virginia, 1991-92

Dr. Hiroshi Ito, George Washington University, Washington, DC 1991-92 


\title{
Curricula Vita of Faculty
}

\author{
MANOJ K. BANERJEE \\ Professor of Physics
}

Education:

B.Sc.

Patna University, India

1949

M.Sc.

Calcutta University

1951

Ph.D.

Calcutta University

1956

Experience in Higher Education:

$\begin{array}{lll}1952-57 & \text { Saha Insitute of Nuclear Physics } & \text { Lecturer } \\ 1955-57 & \text { Princeton University } & \text { Visiting Fellow } \\ 1957-60 & \text { Saha Institute of Nuclear Physics } & \text { Reader } \\ 1959-60 & \text { Princeton University } & \text { Research Associate } \\ 1960-66 & \text { Sitia Institute of Nuclear Physics } & \text { Professor } \\ 1962-63 & \text { Weizmann Institute of Science, Israel } & \text { Weizmann Fellow } \\ 1966-67 & \text { University of Maryland } & \text { Visiting Professor } \\ 1967- & \text { University of Maryland } & \text { Professor } \\ 1973-74 & \text { University of Manchester, England } & \text { Visiting Professor } \\ 1980-81 & \text { University of Washington } & \text { Visiting Professor } \\ 1987-88 & \text { State Univ. of New York at Stony Brook } & \text { Visiting Professor }\end{array}$

Honors and Awards:

Weizmann Fellowship, 1962-63

Fellow, American Physical Society

Fellow, Indian Academy of Sciences

Former Member, Editorial Board of Physics Letters

Member, Editorial Board of Indian Jour. of Mathematical and Physical Sciences Invited Lecturer, Manchester University, 1989 


\section{THOMAS D. COHEN}

\section{Associate Professor of Physics}

Education:

B.Sc.

Harvard College, Cambridge, MA

1980

Ph.D.

University of Pennsylvania

1985

Experience in Higher Education:

$\begin{array}{lll}1985-87 & \text { University of Maryland } & \text { Research Associate } \\ 1987-88 & \text { University of Maryland } & \text { Asst. Res. Scientist } \\ 1988-92 & \text { University of Maryland } & \text { Assistant Professor } \\ 1992- & \text { University of Maryland } & \text { Associate Professor }\end{array}$

Honors and Awards:

Magna Cum Laude in Physics, Harvard College (1980)

Warner Teusch Award-for excellence and promise as demonstrated by the Ph.D. exam (1981)

University Fellowship (1983-84)

N.S.F. Presidential Young Investigator Award, 1990-95 
JAMES J. GRIFFIN

Professor of Physics

Education:
B.S.
Villanova College 1952
M. S.
Princeton University 1955
Ph.D.
Princeton University 1956

Experience in Higher Education:

$\begin{array}{lll}\text { 1955-56 } & \text { Inst. for Theor. Physics, Copenhagen } & \text { Fulbright Scholar } \\ 1956-65 & \text { Los Alamos Scientific Laboratory } & \text { Theoretical Physicist } \\ 1959-60 & \text { Univ. of Birmingham, England } & \text { NSF Fellow/Postdoc Scholar } \\ 1965 & \text { Spec. Summer Lect. Ser., O.R.N.L. } & \text { Summer Lecturer } \\ 1965-66 & \text { University of Wisconsin } & \text { Visiting Lecturer } \\ 1966-68 & \text { University of Maryland } & \text { Assistant Professor } \\ 1968-73 & \text { University of Maryland } & \text { Associate Professor } \\ 1968-69 & \text { University of Maryland } & \text { Associate Chairman } \\ 1970 & \text { Lawrence Berkely Lab, California } & \text { Vis. Summer Scientist } \\ 1972-73 & \text { John Simon Guggenheim Found., LBL } & \text { Fellow \& Vis. Scientist } \\ 1973- & \text { University of Maryland } & \text { Professor } \\ 1975 & \text { Brookhaven National Laboratory } & \text { Vis. Summer Scientist } \\ 1975-76 & \text { Alex. von Humboldt Found., W. Germany } & \text { Senior U.S. Scientist } \\ 1987-88 & \text { Joint Inst.-Heavy Ion Res., Oak Ridge } & \text { Visiting Scientist } \\ & \text { Los Alamos National Laboratory } & \text { Visiting Scientist } \\ & \text { Inst. for Nucl. Sci., Grenoble } & \text { Visiting Scientist } \\ 1989 & \text { Inst.-Heavy lon Res. (GSI), Darmstadt } & \text { Visiting Scientist } \\ 1991 & \text { ITP \& Technische-Hoschule, Darmstadt } & \text { Visiting Scientist } \\ & \text { CEN, Bordeaux-Gradignon } & \text { Visiting Professor } \\ & \text { CEN, Bordeaux-gradignon } & \text { Visiting Professor } \\ \end{array}$




\section{Honors and Awards:}

Fulbright Scholar, Inst. for Theoretical Physics, Copenhagen,

Denmark, 1955-56

U.S. National Science Foundation Postdoctoral Research Fellow, University of

Birmingham, England, 1959-60

John Simon Guggenheim Foundation Fellow, Lawrence Berkeley Lab., 1972-73

Alexander von Humbolt Foundation Senior Scientist Fellowship, Justus Liebig

Univ., Giessen and Hahn-Meitner-Institute, Berlin, Germany, 1975-76

Distinguished Visiting Lecturer, University of Toronto, Canada,

March 1978 (three lectures)

Invited Lecturer, Organization of American States Summer School,

July 1978 (three lectures)

Invited Lecturer, XI Annual Polish Summer School, Mikolajki, Poland,

September 1978 (three lectures)

Lisited in: American Men of Science, World Who's Who in Science, Leaders in American Science, Who's Who in the East

Distinguished Invited Guest Lecturer of the People's Republic of China, April-May 1980

Invited Lecturer in Select Winter Institute on Nuclear Heavy Ion Dynamics,

The Weizmann Institute, Rehovot, Israel, November-December, 1981

Joint I.P.S.T./Physics Department Research Professor, 1981-83

Invited Lecturer, RCNP-Kikuchi Summer School of Physics, Kyoto, Japan 1983

Guest Scientist of the C.N.R.S., France, May 1984

Author of Citation Classic cited by Current Contents 16, No. 31 (August 1985)

Invited Reviewer of Fifty Years of Fission Theory at Memorial Conf. on

50 Years with Nuclear Fission, N.I.S.T., Gaithershurg, April 1989

Semester Research Award from Univ. of Maryland Graduate School, Fall 1989 
WILLIAM M. MacDONALD

Professor of Physics

Education:

$\begin{array}{lll}\text { B.A. University of Pittsburgh } & 1950\end{array}$

Ph.D. Princeton University 1955

Experience in Higher Education:

$\begin{array}{lll}\text { 1954-55 } & \text { Lawrence Berkeley Lab, California } & \text { Theoretical Physicist } \\ 1955-56 & \text { University of Wisconsin } & \text { Visiting Lecturer } \\ 1956-59 & \text { University of Maryland } & \text { Assistant Professor } \\ \text { 1960-63 } & \text { University of Maryland } & \text { Associate Professor } \\ 1962-63 & \text { Lab Joliot-Curie, Orsay (Seine et Olse) } & \text { NATO Fellow } \\ 1963- & \text { University of Maryland } & \text { Professor } \\ 1968-69 & \text { Theor. Phys., U.K. Res. Estab., Harwell } & \text { Visiting Scientist } \\ 1976-77 & \text { Nat. Bureau of Standards, Gaithersburg } & \text { Visiting Scientist } \\ 1984-85 & \text { Sabbatical Leave, Univ. of Maryland } & \\ 1987-88 & \text { National Science Foundation } & \text { Program Director }\end{array}$

Honors and Awards:

Sarah Mellon Scaife Scholarship, University of Pittsburgh, 1946-50

Sigma Xi Membership, 1949

President of Tau Beta Phi Honor Society, 1949

A. G. Worthing Memorial Award for the Outstanding Physics Student, 1950

Sterling Fellowship, Yale University (declined), 1950

Horace Rackham Fellowship, University of Michigan (declined), 1950

Applied Mathematics Fellowship, Massachusetts Institute of Technology

(declined), 1950 
Summa Cum Laude, University of Pittsburgh, 1950

Fellow, Princeton University, 1952-53

NATO Senior Postdoctoral Fellowship, 1962-63

Fellow of American Physical Society

Listed in: American Men and Women of Science, Who's Who in America, World Who's Who in Science

General Research Board Grant, 1985

National Science Foundation Outstanding Performance Award, 1988

Washington Philosophical Society Membership 
EDWARD F. REDISH

Professor of Physics

Education:

$\begin{array}{ll}\text { A.B. } & \text { Princeton University } 1963 \\ \text { Ph.D. } & \text { Massachusetts Institute of Technology }\end{array}$

Experience in Higher Education:

1968-70

1970-74

1973-74

1974-79

$1977-78$

1979-

$1982-85$

$1985-86$
University of Maryland

University of Maryland

Centre d'Etudes Nucleaires, Saclay

University of Maryland

Goddard Space Flight Ctr., Greenbelt

University of Maryland

University of Maryland

Indiana Univ. Cyclotron Facility
CTP Fellow

Assistant Professor

Visit. Foreign Collaborator

Associate Professor

Resident Res. Assoc.

Professor

Chairman, Dept. of Physics

Visiting Professor

Honors and Awards:

Magna Cum Laude in Physics, Princeton University, 1963

Shiuchi Kusaka Memorial Prize in Physics, 1963

Sigma Xi Physics Prize, 1963

Phi Beta Kappa, 1963

Elected to Sigma Xi, 1963

Honorary Woodrow Wilson Fellow, 1963

N.S.F. Pre-doctoral Fellow, 1963-65, 1966-67

N.A.S.-N.R.C. Senior ResIdent Research Associate, 1977-78

Institute Science Medal, Central Research Institute for Physics (Budapest, Hungary) 1979 
Fellow, American Physical Society, 1983

Fellow, Washington Academy of Sciences, 1988

Leo Schubert Award for the Teaching of Sciences, Washington Academy of Sciences, 1988

Maryland Assoc. of Higher Education, Outstanding Educator Award, 1989

Listed in: Who's Who in the East, Who's Who in Frontier Science and Technology, American Men and Women of Science, Who's Who in Technology Today, Who's Who in Society

Computers in Physics, physics education software contest 1990:

Best Simulation: ORBITS

Honorable Mention Simulation: THERMO

Honorable Mention Utilities: The M.U.P.P.E.T. Utilities

Fellow, American Association for the Advancement of Science, 1991

Glover Medal Award, Dickinson College, 1991 
STEPHEN J. WALLACE

Professor of Physics

Education:

B.S. Case Institute of Technology 1961

M.S. University of Washington, Seattle 1969

Ph.D. University of Washington, Seattle 1971

Experience in Higher Education:

$\begin{array}{lll}\text { 1968-71 } & \text { University of Washington, Seattle } & \text { Predoctoral Research Assoc. } \\ 1971-72 & \text { University of Florida } & \text { Research Associate } \\ 1972-74 & \text { Harvard University } & \text { Research Associate } \\ 1974-78 & \text { University of Maryland } & \text { Assistant Professor } \\ 1978-83 & \text { University of Maryland } & \text { Associate Professor } \\ 1981-82 & \text { National Bureau of Standards } & \text { Visiting Scientist } \\ 1983- & \text { University of Maryland } & \text { Professor } \\ 1988 & \text { Los Alamos National Lab } & \text { Consultant } \\ 1989 & \text { Hebrew Univ. of Jerusalem } & \text { Sheinbrum Visit. Professor } \\ 1989 & \text { University of Utrecht } & \text { Visiting Professor }\end{array}$

Honors and Awards:

Leonard Case Honor Scholarship, 1957

Boeing Company Ph.D. Fellowship, 1968

N.D.E.A.-IV Fellowship, 1970

Visiting Staff Member, Los Alamos Scientific Laboratory, 1974-present

Nominated for Outstanding Young Scientist Award, 1978 and 1979

Joint I.P.S.T./Physics Department Research Professor, 1983-84

U. of Maryland General Research Support Award, 1988-89 
Appointed Donders Chair, Inst. of Theoretical Physics, Univ. of Utrecht, The Netherlands, 1989

Editorial Board, Physical Review C, 1989-91

Fellow, American Physical Society, 1990 


\section{Research Publications}

\section{Published Papers: 1991}

1. SEMI-QUANTITATIVE PHENOMENOLOGY OF COMPOSITE PARTICLE DECAY AND THE $e^{+} e^{-}$PUZZLE, J. J. Griffin, Phys. Rev. Lett. $\underline{6}, 1426$ (1991).

2. RELATIVISTIC PROTON-NUCLEUS SCATTERING AND ONE BOSON EXCHANGE MODELS, K. Maung Maung, F. Gross, J. A. Tjon, L, W. Townsend and S. J. Wallace, Phys. Rev. 4ㅜㄹ, 1378 (1991).

3. EFFECTIVE POTENTIAL OF THE $\triangle$-ISOBAR IN A BACKGROUND SCALAR FIELD, D. K. Griegel, Phys. Rev. 'C43, 904 (1991).

4. COVARIANT FEYNMAN RULES AT FINITE TEMPERATURE:

APPlication to NUClear MatTeR, R. J. Furnstahl and B. D. Serot, Phys. Rev. C443, 105 (1991).

5. IN MEDIUM PROTON-NEUTRON MASS DIFFERENCE AND THE SYSTEMATICS OF THE NOLEN-SCHIFFER ANOMALY, T. D. Cohen, R. J. Furnstahl and M. K. Banerjee, Phys. Rev. C43, 357 (1991).

6. EARLY STAGES OF NUCLEUS-NUCLEUS COLLISIONS: A MICROSCOPIC CALCULATION OF THE INITIAL NUMBER OF DEGREES OF FREEDOM, N. Cindro, M. Korolija, E. Beták and J. J. Griffin, Phys. Rev. Lett. 66, 868 (1991).

7. ANGULAR MOMENTUM PROJECTION WITH QUANTUM MESONS, C.-Y. Ren and M. K. Banerjee, Phys. Rev. C4프, 1981 (1991).

8. ISOVECTOR CURRENT OF $N-N$ POTENTIALS AND PAULI-FORBIDDEN S'TATES, T. E. Kiess and E. F. Redish, Phys. Rev. C43, 2509 (1991).

9. EXOTIC HIGH-ISOSPIN BARYONS IN THE SKYRME MODEL: EXPERIMENTAL OBSERVABLE OR LARGE- $N$ ARTIFACT? T. D. Cohen and D. K. Griegel, Phys. Rev. D43, 3089 (1991). 
10. QUADRONIUM: UNRAVELLING THE $e^{+} e^{-}$PUZZLE, J. J. Griffin, Int. Jour. Mod. Phys. A6, 1985 (1991).

12. OFF-SHELL EFFECTS IN ELASTIC SCATTERING OF PROTONS BY NUCLEI, N. Ottenstein, E. E. van Faassen, J. A. Tjon and S. J. Wallace, Phys. Rev. $\underline{\mathrm{C} 43}, 2393$ (1991).

13. SENSITIVITY OF THE T-MATRIX TO DEFECTS IN THE POTENTIAL, L. Canton and G. Rawitscher, J. Phys. G: Nucl. Part. Phys. 17, 429 (1991).

14. CHARGE DENSITY DIFFERENCES NEAR ${ }^{208} \mathrm{~Pb}$ IN RELATIVISTIC MODELS, R. J. Furnstahl and C. E. Price, Phys. Rev. C444, 895 (1991).

15. BOOST, RECOIL AND WIGNER ROTATION EFFECTS ON NO-PAIR ANALYSES OF PROTON ELASTIC SCATTERING, J. A. Tjon and S. J. Wallace, Phys. Rev. C.44, 1156 (1991).

16. FROM QCD SUM RULES TO RELATIVISTIC NUCLEAR PHYSICS, T. D. Cohen, R. J. Furnstahl, D. K. Griegel, Phys. Rev. Lett. 67, 961 (1991).

17. COVARIANT FEYNMAN RULES AT FINITE TEMPERATUE: A PATH-TIME FORMATION, R. J. Furnstahl and B. D. Serot, Phys. Rev. C44, 2141 (1991).

18. CONFERENCE SUMMARY, E. F. Redish, in Spin and Isospin in Nuclear Interactions, ed. S. W. Wissink, et al. (Plenum Press, New York, 1991) 503.

19. RELATIVISTIC AND OFF-SHELL EFFECTS IN ELASTIC PROTON SCATtering, S. J. Wallace, Proc. 2nd Int. Conf. on Medium- and High-Energy Nuclear Physics, Taipei, Taiwan, May 1990 (World Scientific, Singapore, 1991).

20. THE COMPOSITE PARTICLE (QUADRONIUM?) SCENARIO: UnRavelling THE $\left(e^{+} e^{-}\right)$PUZZLE, J. J. Griffin, Proc. 6th Int. Conf. on Nuclear Reaction Mechanisms, Varenna, Italy, June 1991, ed. E. Gadioli (U. of Milana, 1991). 
21. RELATIVISTIC NUCLEAR PHYSICS, S. J. Wallace, in Structure of Hadrons and Ifadronic Matter, eds. O. Scholten and J. H. Koch (World Scientific, Singapore, 1991), pp. 84-113.

\section{Published Papers: 1992 (January-May}

1. COMPOSITE NUClEONS AND THE DIRAC SEA, T. D. Cohen, Phys. Rev. $\underline{\mathrm{C} 45}, 333$ (1992).

2. NUCLEON IN NUClear MATteR, M. K. Banerjee, Phys. Rev. $\underline{\text { C45 }}, 1359$ (1992).

3. TRITON WITH N-N POTENTIAL FROM A QUARK MODEL, S. Takeuchi, T. Cheon and E. F. Redish, to be published in Phys. Lett. (1992).

4. DYNAMICAL CHIRAL RESTORATION IN DENSE MATTER IN HYPERSPHERICAL UNIT CELLS, H. Forkel, to be published in Phys. Lett. B (1992).

5. PASSAGE OF HIGH ENERGY PARTONS THROUGH A QUARK-GLUON PLASMA, Y. Koike and T. Matsui, to be published in Phys. Rev. D (1992).

6. QUARK AND GLUON CONDENSATES IN NUCLEAR MATTER, T. D. Cohen, R. J. Furnstahl, and D. K. Griegel, Phys. Rev. C45, 1881 (1992).

7. AUTHOR'S REPLY TO THE COMMENT ON "COMPOSITE PARTICLE DECAY AND THE $e^{+} e^{-}$PUZZLE", J. J. Griffin, Phys. Rev. Lett. $\underline{66}, 1960$ (1992).

8. THREE-BODY COLLAPSE FOR TABAKIN POTENTIALS AND THE THOMAS EFFECT, A. Delfino, S. K. Adhikari, L. Tomio and T. Frederico, to be published in Phys. Rev. C (1992).

\section{Invited Papers: 1991-92}

1. RELATIVISTIC NUCLEAR PHYSICS, S. J. Wallace, in Structure of Hadrons and Hadronic Matter, eds. O. Scholten and J. H. Koch (World Scientific, Singapore, 1991), pp. 84-113. 
2. CONFERENCE SUMMARY, Edward F. Redish, in Spin and Isospin in Nuclear Interactions, Int. Conf. on Spin and Isospin in Nuclear Reactions, Telluride, Colorado, March 1991, ed. S. W. Wissink, et al. (Plenum Press, New York, 1991) 503.

3. THE COMPOSITE-PARTICLE (QUADRONIUM?) SCENARIO UNRAVELling THE $\left(e^{+} e^{-}\right)$PUZZLE, J. J. Griffin, Proc. 6th Int. Conf. on Nuclear Reaction Mechanisms, Varenna, Italy, July 1991.

4. QCD AND LOW ENERGY NUCLEAR PHYSICS, M. K. Banerjee, Int. Conf. on Medium and High Energy Nuclear Physics, Calcutta, India, Dec. 1991.

5. A PEDESTRIAN'S GUIDE TO LARGE $N_{c}$ ANALYSIS, M. K. Banerjee, II Workshop on Hadron Physics, Sao Paulo, Brazil, April 1992.

Contributed Papers: 1991-92 (Published in Proceedings or Books)

1. OFF SHELl AMPlitudes at NEGATIVE ENERGies, A. Delfino and E. F. Redish, Few Body XIII: 13th Int. Conf. on Few Body Problems in Physics, Adelaide, Australia, Jan. 1992.

2. THE CONNECTION BETWEEN THE THOMAS AND EFIMOV EFFECTS, A. Delfino and

E. F. Redish, Few Body XIII: 13th Int. Conf. on Few Body Problems in Physics, Adelaide, Australia, Jan. 1992.

3. THREE-BODY BOUND STATE COLLAPSE RELATED WITH THOMAS EFFECT, T. Frederico, L. Tomio, A. Delfino and S. K. Adhikari, Few Body XIII: 13th Int. Conf. on Few Body Problems in Physics, Adelaide, Australia, Jan. 1992.

4. VIABILITY OF COMPOSITE PARTICLE SCENARIOS FOR THE $\left(e^{+} e^{-}\right)$-PUZZLE, J. J. Griffin, 1992 Int. Nuclear Physics Conf., Weisbaden, Germany, 1992. 


\section{Papers Submitted for Publication}

(as of May 1992)

1. POWER FALLOFF OF PION FORM FACTOR, L. S. Kisslinger and S. W. Wang, U. Md. PP\#91-233, DOE/ER/40322-122 (1991), submitted to Nucl. Phys. B.

2. INTERPRETATION OF THE GLUON CONTRIBUTION TO THE PROTON SPIN CONTENT, M. K. Banerjee and T. D. Cohen, U. Md. PP\#91-267, DOE/ER/40322-125 (1991), submitted to Phys. Rev. D.

3. $\Delta L=2$ PROCESSES AND PHYSICS BEYOND THE STANDARD MODEL, L. S. Kisslinger and R. N. Mohapatra, U. Md. PP\#92-021, DOE/ER/40322-133 (1991), submitted to Phys. Rev. D.

4. HEAVY-HIGGS BOUND STATE AND BOOTSTRAP, G. Rupp, U. Md. PP\#92-031, DOE/ER/40322-146 (1991), submitted to Phys. Rev. Lett.

5. SPLITTING OF NEUTRON AND PROTON ELECTRIC POLARIZABILITIES IN A CHIRAL SOLITON MODEL, W. Broniowski, M. K. Banerjee, T. D. Cohen, U. Md. PP\#92-130, DOE/ER/40322-144 (1992), submitted to Phys. Lett. B.

6. PROBING VECTOR MESON PROPERTIES IN NUCLEI WITH PHOTOPRODUCTION OF THE NEUTRAL PION, E. Smith-Rowland and T. D. Cohen, U. Md. PP\#92-165, DOE/ER/40322-150 (1992), submitted to Phys. Lett. B.

7. PION LOOPS IN QUENCHED QCD, T. D. Cohen and D. B. Leinweber, U. Md. PP\#92-189, DOE/ER/40322-152 (1992), submitted to Phys. Lett. B.

8. CHIRAL CORRECTIONS TO LATTICE CALCULATIONS OF CHARGE RADII, D. B. Leinweber and T. D. Cohen, U. Md. PP\#92-190, DOE/ER/40322-153 (1992), submitted to Phys. Rev. D.

9. DECUPLET BARYON STRUC'TURE FROM LATTICE QCD, D. Leinweber, T. Draper and R. M. Woloshyn, U. Md. PP\#92-188, DOE/ER/40322-151 (1992), 
submitted to Phys. Rev. D.

10. RESPONSE OF NUCLEON TO EXTERNAL PROBES IN HEDGEHOG MODELS: I. ELECTROMAGNETIC POLARIZABILITIES, W. Broniowski and T. D. Cohen, U. Md. PP\#92-193, DOE/ER/40322-255 (1992), submitted to Phys. Rev. D.

11. RESPONSE OF NUCLEON TO EXTERNAL PROBES IN HEDGEHOG MODELS: II. GENERAL FORMALISM, W. Broniowski and T. D. Cohen, U. Md. PP\#92-225, DOE/ER/40322-163 (1992), submitted to Phys. Rev. D.

12. QCD SUM RULES FOR NUCLEONS IN NUCLEAR MATTER, R. J. Furnstahl, T. D. Cohenand D. K. Griegel, U. Md. PP\#92-219, DOE/ER/40322-162 (1992), submitted to Phys. Rev. C.

Theses: 1991-92

1. STUDY OF CHIRAL CONFINING MODEL WITH VECTOR MESONS, Ching-Yun Ren (adviser: M. K. Banerjee).

2. NUCLEON PROPAGATION IN NUCLEAR MATTER: A QCD SUM RULE APPROACH, D. K. Griegel (adviser: T. D. Cohen). 

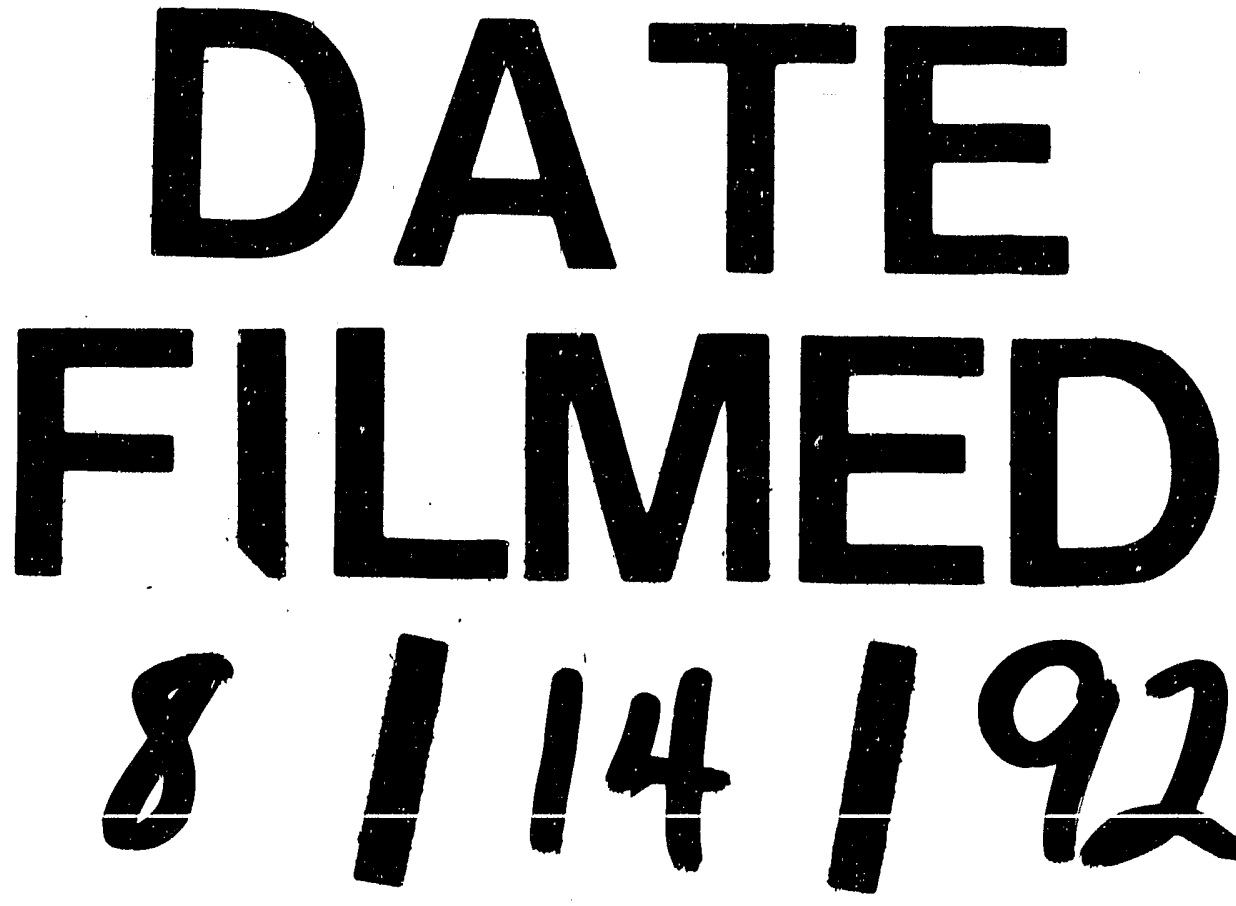

14
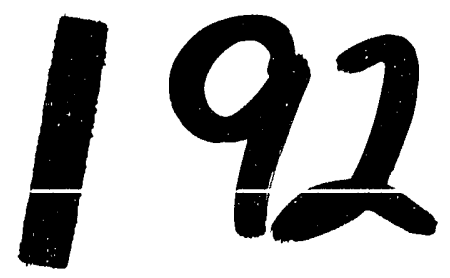


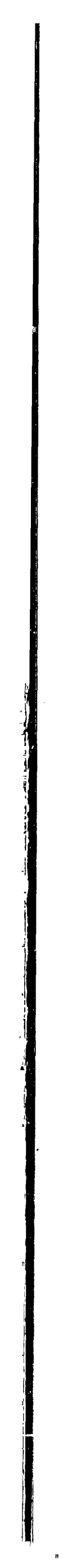

